\title{
Centralized Connectivity for Multiwireless Edge Computing and Cellular Platform: A Smart Vehicle Parking System
}

\author{
Aamir Shahzad $\left(\mathbb{D},{ }^{1}\right.$ Jae-young Choi $\mathbb{D}^{2},{ }^{2}$ Naixue Xiong $(\mathbb{D})^{3}$ \\ Young-Gab Kim (D), and Malrey Lee ${ }^{1}{ }^{4}$ \\ ${ }^{1}$ Department of Computer and Information Security, Sejong University, 209 Neungdong-ro, Gwangjin-gu, \\ Seoul 05006, Republic of Korea \\ ${ }^{2}$ Department of Computer Engineering, Sungkyunkwan University, Suwon 16419, Republic of Korea \\ ${ }^{3}$ Department of Mathematics and Computer Science, Northeastern State University, 611 N. Grand Ave, Tahlequah, OK 74464, USA \\ ${ }^{4}$ Center for Advanced Image and Information Technology, School of Electronics \& Information Engineering, \\ Chonbuk National University, 664-14 1Ga Deokjin-dong, Jeonju, Chonbuk 561-756, Republic of Korea
}

Correspondence should be addressed to Young-Gab Kim; alwaysgabi@sejong.ac.kr and Malrey Lee; mrlee@chonbuk.ac.kr

Received 13 October 2017; Revised 2 December 2017; Accepted 17 December 2017; Published 20 February 2018

Academic Editor: Shangguang Wang

Copyright (c) 2018 Aamir Shahzad et al. This is an open access article distributed under the Creative Commons Attribution License, which permits unrestricted use, distribution, and reproduction in any medium, provided the original work is properly cited.

\begin{abstract}
This study takes an intuitive step to develop the user-convenient smart vehicle parking system (SVPS), a smart system able to manage the massive crowd of vehicles during parking searching and do the better jobs of parking reservation and management, with the shorter-path processing tactics. For that, this study inclusively employed the mapping strategy, where the system parking points are prevalent, to assist the users to get the parking information fast and conveniently. This study is comprised of the several parking points systematically spread over the several locations and traceable over the available graphical map, and the overall information is easily accessible using smart devices. For parking information, a smart web application which is another important module of this study is designed, with which the SVPS system's registered users are able to access all the services provided for smart vehicle parking searching and reservation in efficient and reliable ways. An integrated network approach, RFID (radio frequency identification) and wireless sensors network (WSN), called RF-WSN, is employed to retrieve the real-time information from the installed and configured sensor devices in RFID-WSN network.
\end{abstract}

\section{Introduction}

With the enhancements of the Internet of Things (IoT) that have been made in several areas of human lives, the traffic control and management systems are predominated, and for that, several intelligent solutions are implemented to manage the massive traffic crowd in metropolitan areas. Typically, a long time ago, the human movements are massively increasing towards the big cities, in almost all over the world; therefore the traffic issues are also increasing at the same time more especially in the cases of finding the parking places. Notably, the case of finding a parking place in a cowed city that has available parking lots is a big challenge, which has been faced by vehicles owners. The recently approaches employed to find the vehicle parking lots are manual; therefore the case of finding parking lots in the metropolitan city in a massive traffic crowd is a big task. Some parking places used computerized systems to keep the checks on vehicles that are going in/out from the parking places and monitoring systems are mainly facilitated through the surveillance systems (e.g., CCTV). However, it depends on the person; if he/she is lucky, he/she could find the parking space upon arrival at the parking place; thus this is very transparent that most of the time is always wasted in finding parking place, if available. Also, in most cases, the vehicle's owner always finds the parking place far from his/her destination; consequently this is not an optimal approach, even in case of predefined parking, which wastes time and vehicle fuel and other energy consumptions [1-3]. For enhancement through the employing of modern technologies, such as the 
Internet, wireless, and cellular communications, numerous interactive and noninteractive systems have been introduced for vehicle parking reservations online before the time to search for the destination. This is somehow good but there is no surety to get the confirmation, through the cellular devices, of parking place at the same when required $[1,4,5]$.

The smart parking system is also considered as a part of the smart city project, where the advanced technology such as the Internet of Things has been ubiquitously deployed in various sectors, to make the user lives convenient by providing their required services. Thereby, through its use, users or vehicle drivers can able to find a parking lot in any part of the city and will reserve the vacant parking lot according to their predilections, through Internet connectivity, using of electronic reachable devices or/and cellular devices. The parking fees possibly are made using debit/credit cards at the same parking place using parking payment machines. Accessing through the Internet, the smart online parking applications, or parking systems, the user should precisely get the all available information of parking place, parking spots availabilities, parking reservation upon decision, and fees detail and reliable payment methods; of course, it saves enough time and avoids the massive traffic congestion issues from user perspectives. Moreover, during parking spot selection and at the time of car parking, car management system is also a main part of smart parking system which is deployed efficiently to manage the tasks such as online accessible parking point localization, parking reservation through Internet access, vehicle security through tracing the networked tags, parking barrier control, RFID based parking payments, and others [6,7]. Usually, two types of management systems, (1) main parking gate tracking system and (2) parking lot tracking system, are addressed under smart parking system. The cars (or other vehicles), during entry and exit, passing by the main parking gate, at each check (checkin or checkout) are observed and managed which resulted in various important services to the drivers, including the availability of vacant parking lots in a parking place and availability to make a reservation online, whereas the presence and absence of each car parking over the indicated parking lot are monitored through reading from installed sensors at that lot. Parking lots are generally equipped with sensors or camera equipment for lot monitoring and processed and managed by lot management module as a part of the smart parking system, which therefore also provides services to their users (vehicle owner) including the lot availability and where to park map guideline. Moreover, the parking lot management module can be varying to parking lot design and area, such as indoor lot design (or monoparking) and outdoor lot design; then selective parking lot's module is addressed to manage based on reading observed via lot's sensor installed [6].

Radio frequency identification (RFID) is commonly employed innovative technology, as an important part of the wireless communication system deployed in various automotive industries, aviation and medical sectors and transportation, and parking management systems and others. The commonly used equipment, which made the RFID transmission through radio waves, is the RFID tags (such as active and passive), tags reader, communication media, computer system encompasses of databases, user interface, middleware, gateway, and so forth [7, 8]. Therefore, the RFID technology has been considered tremendous due to facts of cost reduction and gain system's computation efficiency, deployed in a wide range of technological sectors and applications, employing a specific set of hardware and the proprietary protocols, in order to make communication to reading data accurately from tags possible for RFID reader. In RFID system, the two types of tags are commonly used. (1) Active tag, containing an embedded IC chip, coiled antenna, and built-in power source, carries the low-level energy signals from RFID reader and then transmits the identification or resulted signals back to the reader. (2) Passive tag, another type of RFID system in which tag does not have an internal power source and its use of the energy source of RFID reader usually designed to transmit the high power signal towards an RFID tag, uses same power even to transmit back the modulated signals required. The RFID reader is the main module, used in RFID technology, which spreads the signal around in order to get the information from the tag; however, reader is able to get modulated signals from multiple tags at the same time and then process to the application program running on the system for further processing, including storage. In case of an active tag, the identification signal is transmitted around and continued powering with/without concerning reader's field signals, whereas passive tags are activated while high-level signal is received from RFID reader [7-9]. In practical, RFID technology has been playing rigorous roles and widely developed for the transportation systems, in which the vehicle identification in parking systems is more dominated $[6,10]$.

In automated parking systems, the RFID technology has important roles in vehicle identifications and automated parking fee payments; RFID system enables the autofast and efficient and secure monitoring during the vehicle checkin and checkout from parking gates (or barriers) through tracking tags from RFID reader, including the gates' controlling and their timing that is specified, in the parking place. Therefore, the vehicles can move in/out from the parking gate in sequential order, with the minor delay, which avoids the congestion issues and the multiple vehicle checks-in and checkouts, and the parking payments are collected without any delay that may require each vehicle to stop at the parking gate, through using of RFID based ticket machines $[7,11]$. The RFID is a contactless technology employed for object tracking identification, communicating through radio signals, and thus most appropriate for vehicle parking system due to its several benefits: (1) contactless physical communication, (2) enabling tags placement in a loop, possibility of using even in worst scenarios, and environmental conditions, (3) tags which are available according to the communication system demands in the wide range and sizes, (4) minimal service costs, and (5) minimal error rate in nonline of sight communication and others. For an optimal parking system, RFID base system considered as a solution to well manages the parking lots management issues encountered in existing studies [12]. The major issue, such as application processing issue, when the parking lots are not available, resulted in the parking space management issue during the 
application process. This issue could be resolved by keeping out those vehicles which are supposed to check in; however, enough time will probably be spent on searching the parking vacant lots. Another major issue generally happened during short/long disconnection with the main system via Internet access, and these issues can be resolved by keeping the communication continued, perform each transaction, and store in the local system setup updated all the saved data that are recorded, to the main system's storage, while the main system status changed to online. Thus, this is a good approach employed to run also the database on a local system and further shift all information to the main system upon connection established with [11].

In studies [10, 12-16], comprehensive reviews were conducted on the smart parking systems which deployed the today's advanced IoT technology; further some important issues were highlighted that were commonly available in parking systems and the usual challenges that have been faced by the drivers (or users) during searching the optimal ways for the parking in the traffic density in the crowded areas. More advanced, in study [10], smart parking system was built with the mathematical model formed to validate the overall system's performance and parking reservations were performed with the minimal cost, but, at the same time, this study has some disadvantages, including the time wastage consumed during car forwarding scenarios to another parking place. Therefore, here, smart parking system needs special care and acquires efficient solution to resolve the car forward issues to the other parking areas, before the case where the researched parking status is full.

In this proposed study, an inclusive and convenient approach is precisely planned to deploy the automated smart vehicle parking system (SVPS), and the SVPS system is intelligently designed and modeled as a complete solution to regulate and monitor its parking points and other pieces of networked equipment and is considering under public sector regulations. In short, this means that the whole proposed system is directly associated with the public sector or governmental sector property. Therefore, the system development emerges and plays important roles in economic benefits and benefits for the users to get the convenient and cheap parking while comparing the rates with the private sector parking systems. Following are the main objectives this study aims to fulfill (or anticipate):

(1) SVPS system is only accessible for its users; therefore a registration module is designed and available online which facilities the registration process for the new users. Upon registration completion, the users could get the RFID tag installation, as well as the RFID access card and the online login ID and the password.

(2) Upon logging in to the online SVPS system, the parking searching and reservation module is designed, as a part of SVPS system's web application, to made parking search easy using designed mapping strategy and to made parking reservation according to user selection or user request for parking. Through mapping, users will find the near parking points and the short route to reach the destinations. Further, the system is efficient in computing the short travelling route, followed by the input location of the user.

(3) At time to access the parking point and selective parking lot, the embedded RFID tag and designed RFID access card are used in order to access main parking gate. For user (or vehicle) authentication and verification, at parking point entry gate the RFID tag is read and the RFID card is verified wirelessly through installed sensors. Then, one more time, vehicle embedded RFID tag is read at its allocated parking lot, for parking lot verification purposes.

(4) For parking utilization and the corresponded payable amounts, the SVPS system is smart in order to show the overall payment of parking utilized, at per hour basis, online into the user account and the parking payment would possibly be payable using various payment services (i.e., using credit/debit card and monthly based transferring facility). Therefore, the user has no worries about the payment to be made at the time of parking (or parking exit).

In this study, the parking point (PP) is used to represent the parking place where a number of parking lots $(\mathrm{PL})$ are available for vehicle parking and the registered vehicles are represented as users of the system. Moreover, in RFID-WSN system, the installed RFID UHF readers at parking lots are designated as slave-sensor nodes connected with the mastersensor nodes.

The rest of paper is organized as follows: Section 2 conducted a comprehensive survey on the various solutions, systems, and technologies employed in existing vehicle parking systems. The system design and framework are described in Section 3 which with the study fulfills its target goals, and in Section 4, smart parking design is detailed and then set up with the numerous of sensor nodes. Section 5 implements a system where registered users or users of the system are allowed to get access the smart parking services available as part of the system and do the parking reservations. Experimental scenarios are defined and employed to conduct the results of the discussion in Section 6. At the end, Section 7 concluded the overall study works and the directions for future work.

\section{Literature Survey}

Smart parking system, employing Internet of Things (IoT) technology, was implemented to facilitate the parking reservations [2], accessed through the online parking service, using the cellular devices and other accessible devices like tablets, and further the end-users were be able to see the availability of parking lots before the reservations. As a solution, a valid unique identification number (UID) was assigned to each vehicle and used during the whole online parking reservation process and that number should be further applied to authenticate the vehicles at the main entrance of parking place, using RFID devices installed $[2,7,17]$. In spite of, by considering, notably, the limitations of the existing vehicle parking systems, such as undefined short distance parking, managing of traffic load balancing, instantaneous 
query acceptance and rejection, and commercial welfare, the IoT technology has been playing important roles to resolve these issues. In a study [1], a cloud-based smart car parking system is modeled, considering the efficient computations of IoT technology, in which each parking place is deemed as the IoT communication node. The important required information is that the vehicle positions' computation using global positioning system (GPS), the estimation of the distance between the current position of the vehicle and the closed parking places, and parking spaces' availability are examined in real-time manners. The observed information is then simultaneously transmitted back to the cloud data center, where the carried information is stored, managed, and updated and is further accessible by the authorized user's request. For system prototyping, physical IoT Arduino platform is used, and the parking places are fully equipped with the RFID technology [1]. A short message service (SMS) is used, as an intelligent parking solution, considering hardware design, using the TC35i module in a global system for mobile communication (GSM), which avoids staying at other wireless media because of the development cost. Thus, using this service, a message sent by the driver to the system to check the status of the parking lots in the parking area and upon confirmation of available parking, the driver allowed using the parking lot as long as the time mentioned, inside the SMS received from the system [18].

In studies [18-21], the advanced technological frameworks for smart car parking systems are introduced, employing the hybrid network, in wired and wireless sensor networks, which deployed modern RFID technology and IEEE standards for communication. Further, the developed smart parking systems are accessible online using Internet's facility available. Thus, upon the parking reservation and confirmation, the system collects the information of available parking places and the information of vacant lots and transmitted it back to the users who could see parking information, using the smart parking application installed in their smart devices [7, 11]. While entering the assigned parking place, the system's allocated user parking lot would appear on the small displaying parking map, employing a short-range protocol called dedicated short-range communications (DSRC). DSRC protocols have been employing several intelligent transportations systems [22]. Further, in the boundaryparking place, inertial navigation system (INS) has been commonly deployed to direct the vehicle to its originated parking position. Thereby, the system design becomes more efficient and gains high accuracy by the means to make parking system always updated, with the information of parking lots, for example, whether vacant or nonvacant, in a specific parking place, in a real-time manner; thus the time usually required by the users to perform parking reservation would significantly minimize [13]. In another study [19], a new architecture, intelligent parking assistant (IPA), is implemented to manage the public parking points located in various parts of the city. The designed architecture is intelligent in computing information of available parking lots in the street parking areas; thus the drivers, those submitted the request for parking, could see the available parking lots information before few minutes of their arrival to the selective parking points. The IPA design was mainly targeted to manage the parking spot that occupied small coverage area and to keep the vehicle check-in and checkout information always updated in the control unit, through employing of the RFID technology. The RFID devices and the magnetic loops are installed in the parking spots and the IPA gets the parking overall information and keeps the control unit updated, periodically.

In [23], the common car parking issues are considered and then the smart car parking system is proposed, employing of ZigBee network, based on IEEE 802.15.4 specification, suited for personal area networks. In the designed system, a coordinator device establishes the network root and the application layer transmits information quickly back to the controller via Internet, where the information is kept updated in the database. As a part of system's design, web service is created, a useful service, to keep the parking prevalent information always updated, for the newer users (or drivers), to make the reservations for the parking lots if available. Lambrinos and Dosis [15] introduced an architecture design for smart car parking system, employing advanced platform called the Internet of Things (IoT), the middleware layer, and ZigBee wireless sensor network (WSN), and the required reporting services are manipulated through IoT front-end layer 12 which acts as an interactive user interface. However, the study has limitations, due to the employment of reliable application protocol, that is, constrained application protocol (CoAP), in transferring of information from the network setup to the control unit. Bonde et al. [24] proposed a miniature model for automated car parking system, whose designed aim is to control and manage the availability of parking slots for the cars in the parking place, according to the given allocated times. For this, a software application is designed, based on Android platform, which manages the given allocated time to park the cars and the existing time from occupied parking lots. The sensor devices installed at parking entry points regulate the check-in and checkout to/from the individual parking lot. To check the parking lots information, convenient for the users, an LCD integrated with the microcontroller is placed at the parking entry point, which displays the parking lots status, time to time. In [25], a mechanical model is designed for smart parking system comprised of various parking levels and the lifting facility therefore is used to make parking automatic. A recognition system is deployed, which recognizes the cars at the parking place through their license number plates. For vehicles lots detection, inside parking place, ultrasonic sensors are installed with liquid crystal display to visualize the vacant parking lots status $[26,27]$.

In studies [7, 28-39], numerous automotive vehicle parking systems are designed and modeled according to the time-ongoing requirements of vehicle parking, employing of RFID and wireless sensor networks. For that, sensor devices or sensors mostly comprised into intrusive and the nonintrusive have been employing depending on the constraints, as ubiquitous deployments, such as finance, scalable design area, system reliability, and efficiency, to keep a smart check and balance for the system's usage and processing. Despite intrusive sensors technology, nonintrusive sensors utilization 
in various wireless sensor networks is accounted as a costeffective and easy-to-install solutions, specifically for the video image processing solutions which are more robust compared with the others and are used also in the parking systems to detect the vehicles within the boundary of the parking place. The vehicle detection is carried out using the visual images, as a part of the video image processing system and thus, successful observations (e.g., changes) are made through regulation and comparing the successive frames captured in specific time interval $[26,40]$. More advanced various existing car parking issues are considered and after, image processing based solutions are proposed to facilitate the patrons well [26, 41]. For development [26], "RabbitCore ${ }^{\circledR}$ Microcontroller image processing" unit is used, integrating with the closed-circuit television (CCTV), in the designed parking area. The purposes of the integration are to made detection of the parking lots in the parking area and to transmit the collected information (of vacant parking lots) back to the central server, keeping the information updated in the database, through ZigBee wireless sensor network (WSN). The visual facility, as a part of car parking system, is installed at the main entrance of parking and visualizes the complete parking map, the availability of lots of each level, and the convenient shorter-path direction by the implementation of A-Star $\left(\mathrm{A}^{*}\right)$ algorithm. Automated ticketing and payment machines, located in different parts of parking boundary, are used during entry at the parking and upon exit from the parking place [42]. In [43], the authors propose an image processing solution, deployed to collect the empty car parking spaces information in car parking area. This proposed solution is used to capture the brown rounded image of each parking space and then further performed the detection for free (or available) parking spaces visualize in the seven-segment display. In another study [44], a mechanism is proposed based on the camera vision technique; the free parking lots are detected by setting the input values, for example, input coordinates of parking point, to the object classifier. For that, cars' images are captured in the parking point, through considering various angles, and then classified as positive and negative images. The positive images are used to examine the car presences and on the other side, the negative images are analyzed to show vacant parking or parking lot available for car parking.

\section{Proposed System: Design and Framework}

In this study, the main goal is to provide a fully automated smart vehicle parking system (SVPS) which should significantly reduce the workforce, usually required in conventional-existing parking systems, and aims to employ an efficient way to deploy the radio frequency identification (RFID) technology in wireless sensors network (WSN). The WSN configuration provides the potential benefits of the proposed parking system or SVPS system which showed a new development to fulfill the advanced parking system requirements, which are probably required by today's modern smart parking systems [5, 9-12]. Therefore, this study contributed well in achieving its goals; the development is mainly a consideration as a part of the Internet of Things (IoT) platform, where all the system devices are networked as fully automotive devices have self-controlling abilities to interact with the overall system-modules. For that, the SVPS system is comprised of the modules designed and deployed as services offered by the SVPS system: (1) parking appointment module, (2) registration module, (3) installation module, (4) websearching module includes parking reservation management and parking lot management, and (5) parking design and setup, to fulfill the objectives of proposed study.

In Figure 1 the SVPS system's framework is comprised of four main layers: (1) hardware-sensor layer, (2) network access layer, (3) middleware layer, and (4) user application layer, employed to provide a 2-way communication and the interaction between the SVPS system's modules. More precisely, in the below subsections, the SVPS system definemodules are thoroughly explained followed by these four layers. At the instance, SVPS system's design is entirely computation based on the efficient simulation design, as well the conducted measurements, but is very flexible in the planning of further system extension and system modularity. Further, the SVPS system is a system, absolutely developed, under the regulations of the public sector (or local city governmental level), because of the following:

(1) In future, SVPS system's design and framework will probably take over and regulated the public sector and the further intentions in mind to extend the project as a country-level project.

(2) SVPS system's design and framework are directly/indirectly considered as part of IoT system, that is, IoT ubiquitous smart city project.

3.1. Hardware-Sensor Layer. In SVPS system, at the hardware-sensor layer, incorporating RFID technology and with the integration WSN technology, primarily system's services are stated to perform. Further, importantly, the overall useful information is continuously retrieved from the RFID sensors installed and configured in RFID-WSN system. In Figure 1, SVPS system is comprised of a number of parking points and designed to carry the real-time information (or reading), from the installed RFID sensors, through communicating with gateway system. In each parking point, as a part of SVPS system, the parking lots are installed with RFID readers (or sensors) having specifications as follows: (1) ultrahigh-frequency (UHF) type, the usage available frequency range which is about $860-960 \mathrm{MHz},(2)$ RFID tag detection, the distance range of about 2 meters, and (3) the ISO 18000-6C standard [45-47]. The air-interface protocol (generation 3 ) is employed, a way of communication between labeled passive RFID tags and installed RFID UHF readers. More precisely, the each exploited RFID reader has been assumed to have sensing capabilities while usage of WSN technology, but for instance, the SVPS system employed WSN technology, integrating with RFID technology, only for the purposes of converging or wireless coverage extension. Thereby, incorporating WSN technology, the information from RFID sensors can be forwarded to system gateway installed and set up in each parking point and to the central control system. RFID 

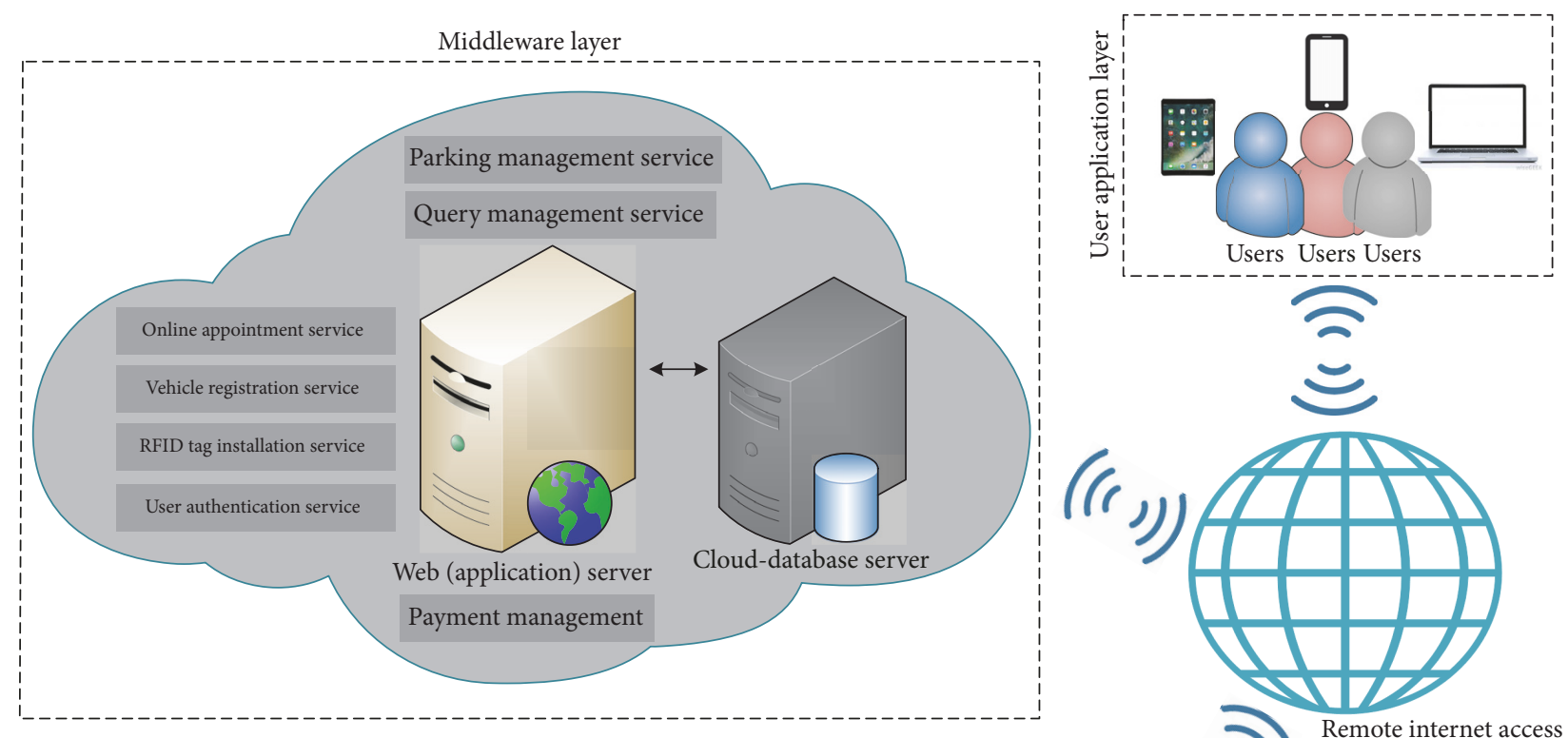

(i) SVPS-PP20: SVPS system PP location 20

(ii) SVPS-PP20-GW: SVPS system PP gateway

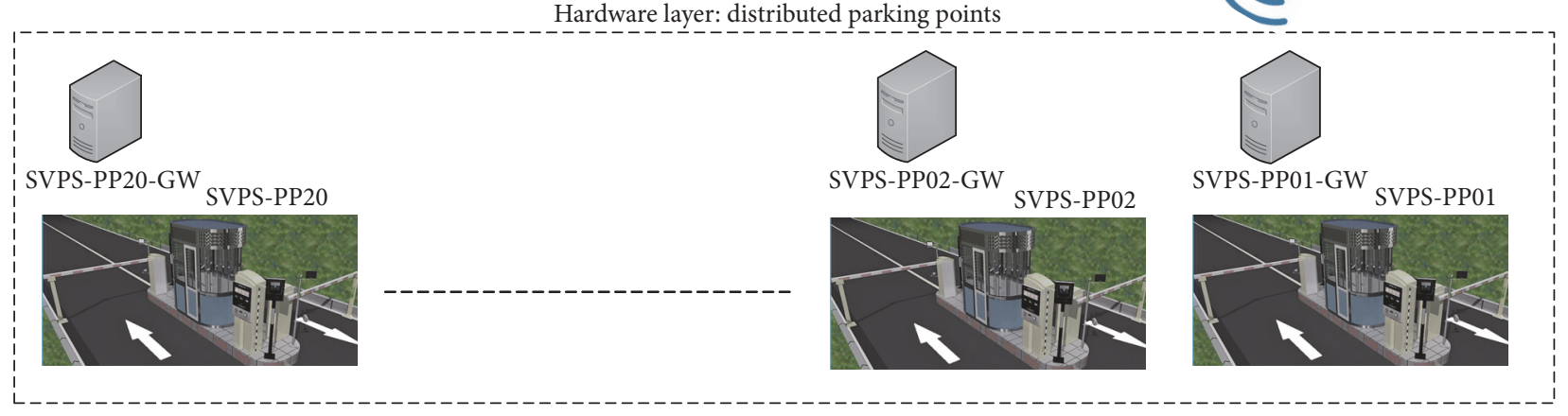

FIGURE 1: System design and framework.

sensors are installed and positioned the exact center of each parking lot, illustrated in Figure 2. The embedded RFID sensors, also called slave-sensor nodes (SS nodes), are connected directly with the master-sensor node (MS node). MS node is an acquisition node configured to retrieve real-time information from the slave-sensor nodes; however, the number of sensors is limited which is assigned to each master-sensor node. Moreover, the SS nodes are connected directly to the MS node through the wired network, and for this, the interintegrated circuit $\left(\mathrm{I}^{2} \mathrm{C}\right)$ protocol is employed which is intended for the communication between slave-sensor nodes and a master-sensor node. For local communication, within the premises of parking point (e.g., personal area network), ZigBee wireless network based on IEEE 802.15.4 specifications is used to access and carry information from networked master-sensor nodes (MS nodes) within the distance range of about 10-100 meters. Further, in RFID-WSN network, the repeaters or the anchor nodes (AC nodes) are networked and distributed over the optimal positions in order to retrieve information and to provide coverage to the master-sensor nodes.
3.2. Network Layer. In network setup of each parking point, the slave-sensor nodes are installed and are directly connected, that is, wired network connection, with the mastersensor nodes designated which are wirelessly connected to the local system controller (LSC). Basically, LSC performs the functionalities of gateway system and in each parking point, LSC is designated to carry the traffic from slavemaster-sensors nodes and to pass the traffic to the central controller system (CC system) using transmission control protocol (TCP)/Internet protocol (IP), a way of transmission over the Internet. Therefore, the users that made register under SVPS system could get the system's access, using smart electronic devices including GSM/GPRS based cellular devices, directly from the CC system. This means, because of the security issues, the external smart devices exploiting the parking reservations and other system available services are only allowed to access through CC system, not from the selective parking gateway system, only from the central system. However, in few situations or critical situations, the users could access information directly from the target parking point gateway system; this is one of the future considerations 


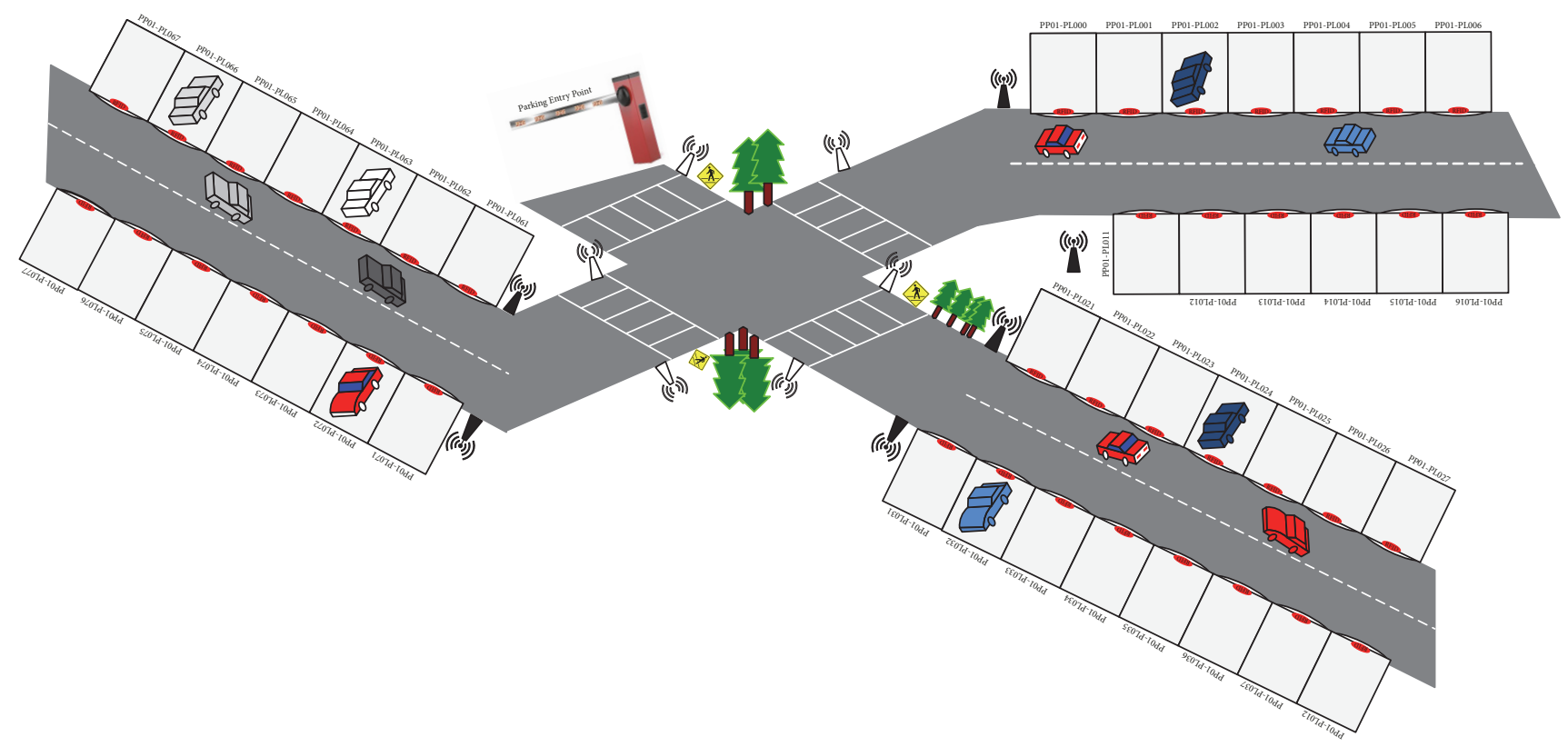

RFID reader/slave-sensor node

Master-sensor node

Repeater/anchor node
}

FIGURE 2: RFID-WSN network setup and configuration.

which will be deployed in presences of security framework that is out of the scope of this study.

\subsection{Middleware Layer}

3.3.1. Development and Database. In this study, the central controller system is considered as a main centralized controller to access, monitor, and control the overall information from/to the remotely networked parking points which are distributive over the various main locations of the metropolitan city. Therefore, SVPS system performs all required operations, such as the management, monitoring, and controlling operations, through a CC system. SVPS system is entirely designed and developed using the development tools: (1) Microsoft Visual Studio C\# as a programming tool, (2) ASP.net for web applications, (3) IIS server for web access, and (4) MySQL database tool. Further, more advanced add-ons are available, for Android and iOS operation systems running on cellular phones, which intended to allow access to the SVPS system online, merely with compatible predominated search browsers such as Microsoft Explorer, Google Chrome, Firefox, and Safari. Thereby, cellular users could able to access the SVPS system online, through compatible browsers, for example, parking slots availability check and parking reservation purposes.

For efficient online searching for parking relevant information and keeping the record always up to date, the cloudcomputing design is also a part SVPS system. Thus, SVPS system incorporated a cloud design or also called SVPS-cloud, using Microsoft cloud platform actively employed to fulfill the overall backup storage requirements of the system. However, now, the cloud-computing facility employed in SVPS system is only limited to keep the information backup, the information that is carried from the parking points. In SVPScloud, the database design is generic in order to store and keep records of the information individually from each module and is efficient in its computing; therefore, the information can be retrieved inclusively as a result that is computed from distinct modules of SVPS system. For example, those users are only permitted to perform parking reservations previously if they are registered under the system, that is, authorized system's users, and further, the requests made for the parking lots are possibly granted if they are vacant. In order to manage the massive connectivity and network traffic, the SVPS system is developed in such a way that a fully accessible system to cellular devices is running Android or iOS systems, via the Internet. Thereby, the registered users are authorized to access and use the SVPS system's services online using of Internet connectivity available inside the cellular devices.

For user convenience, the parking searching is an important feature employed which provides a reliable and fast way to search the parking points from the location or point where the vehicle is positioned. For that, the Google Maps, using Google application program interface (API), is setup and integrated into SVPS system's design in order to acquire on search a user demandable parking point(s). Thus, as a part of this study, the system uses the Google Maps service to pin the exact parking positions and adds a new feature to locate the 
closer parking points, through computing the distance with all the system's available parking points, followed by the user's selection process (i.e., request for parking). As a result, the system will display the most closer (e.g., 3-4 or more) parking points; therefore, the user has options to select the specific parking point among others by his/her interest.

3.3.2. Timing Allocation and Management. The smart system or SVPS system is designed to operate parking allocations, and the parking timing that is required by each vehicle is entirely managed in hourly basis. There is no limitation for the vehicle to fix the time of parking during reservation, that is, the time to leave the allocated parking lot; the SVPS system automatically computes, manages, and notifies the timing, while each authorized vehicle makes entry onto the parking lot and the time of exit, through sensors reading. However, there might be some delay occurring because of the sensors reading, for example, while information is transmitted from sensors to the local system controller (LSC) to central controller system (CC system). For that, SVPS system efficiently measures the timings (e.g., transmission delays) which considerably do not affect the overall system performance. Moreover, entirely, the system is intelligent in order to acquire the timing information, while the vehicle makes entry at the main entrance of selective parking point through sensors data acquisition. For best timing management, enough additional time will be allowed corresponding to the total distance which might be travelled by the vehicle from parking main entrance to the parking lot entrance (allocated). For example, the distance between the parking main entrance and the allocated parking lot computed by the system is approximately 300 meters, which is covered by a vehicle in almost two (or less) minutes, without traffic congestion inside parking point. As a result, the system allocates additional 2 minutes to that vehicle to make entry onto the parking lot. Particularly, the SVPS system is very flexible and efficient in its design, mainly under the requirements of the users request for parking, and it provides the following 3 main features: useful for parking time allocation and management purposes.

(1) Any Time Parking. The registered users have a feature to do parking reservations, accessing the SVPS system online, at any time. For example, on the spot, the user wishes to do parking of his/her vehicle while being closer to any of parking points. By checking the availability of parking lots and then user parking reservation, thus for that selective parking point, the user will allow making entry inside and could stay longer at the parking lot without any time restrictions assign via the system. However, the system manages the timing calculations and the usage of the parking lot, on an hourly basis.

(2) Fixed Time Parking. This smart parking feature allows users to make reservations for parking points, regulated under the system, in case of parking lots availability, prior to the several hours but no more than 48 hours restricted, for a fixed timing. For example, a registered user wishes to make parking reservation in advance, according to the parking point selection, within 48 hours (e.g., Monday at
14:00 pm-22:00 pm). Thus, the system allocates the time session for parking, corresponding to the user selective fixed timing, in advance.

(3) Timed Parking. The users who aim for the parking on the monthly basis use this feature. Thus, the users have the option to do parking reservations, selecting the parking lots, on the monthly basis, and have the option to reserve parking on a daily and weekly basis. For user convenience, the parking reservation is easily to be made just using the online system and by selecting the starting time/day and ending time/day, using the service provided by the year/monthly calendar additionally having a time-date facility.

For the above cases, the CC system is designated to make the reservations, based on the parking lots availability acquired from slave-master sensors, on the users request for parking (RFP). The system is much efficient in its computing, that is, time computing, whenever the vehicle makes entry at the parking main barrier of selective parking point and then at the parking lot allocated from the system. Therefore, the time vehicle arrives at the parking lot; then local system controller (LSC) transmits the information or check-in and checkout status to the CC systems, through the sensors installed at the parking lot.

3.3.3. Registration and Primary Features. The proposed system or SVPS system is designed under the considerations of the fully automated system; for achieving that, SVPS system utilizes the advanced emerging technologies of the current era called the Internet of Things (IoT). Thereby, the system is considered as a fully automated system and reachable to numerous smart devices, such as cellular devices, laptops, and others, using the Internet access. However, the system is not designed with the considerations of security, that is, Internet security as well as the security protection against the potential system's vulnerabilities [48, 49]. So, the comprehensive security design will be a major contribution to this study aimed to draft in nearly future; nevertheless, here in the current stage, this study considers the inclusive smart setup procedure which is useful during the verification process to verify whether or not the vehicle that beings utilizing the parking service is authorized or not. Therefore, in short, a standard new registration method is employed, which will be significant at each stage of parking process (e.g., parking searching and reservation), as an authorized way regulated under SVPS system.

(1) Registration. One of the important steps, among others, is the verification of vehicle being registered under the SVPS system and the authorized owner identification (e.g., valid identification number). At the time of registration, these are basic requirements for each user to be registered; however, a request for SVPS registration could be possible to be made online using of smart appointment module. Therefore, through entering the user complete details, including his/her vehicle details, into the SVPS system, a unique identification number (UID) is generated and then assigned to the vehicle. The UID shows identity of the user authorization and further uses to access the system services overall. 
(2) Installation. Upon completion of registration process, for example, all necessary tasks, the smart RFID passive tag called Tag-SVPS (or T-SVPS) and an RFID base chip card called C-SVPS are generated, having an identical unique identification number (e.g., IS0004641V780012104P561S), and printed under the registered vehicle. For each vehicle, at the time after registration, both the T-SVPS and C-SVPS are RFID based and have a similar identification number which will be used to track and acquire the vehicle valid information during system utilizations. The usage of C-SVPS is twofold: (1) at the parking entry it provides an authentication through sensing the identity using RFID sensor installed at the parking barrier; (2) alternatively, it will be very useful in case the embedded T-SVPS is not readable by RFID sensor, due to the network issues such as hardware issues and interference; thus the system permits that vehicle to make entry inside via $C$ SVPS. However, this situation is not very common and for operation, it requires permission from the CC system.

To make continually interaction with the system, the TSVPS and C-SVPS having similar identification are always useful, while vehicle identification requires field sensors installed in parking points. For instance, 20 parking points are under the design-consideration of SVPS system and are connected centrally with the CC system, but not with each other. Because of the security issues, the SVPS system only exploits a centralized system (or CC system), intelligently set up to control and manage the information from each parking point.

3.3.4. Smart Access. At the time of registration, a short unique user ID is created, along with the password, for each registered user, that is, it authorized the user of the SVPS system, to access the system services online using of various smart devices. Thus, the system is supportive of the smart devices having various network connections, such as Wifi, GSM, 2G, and $3 \mathrm{G}$, to interact with the system's premises. While logging in to the system online, entering the user ID and secure password on the spot system should get the original accurate position of the user (or user's vehicle location information) through getting the measurements of the positional coordinates, and then the observe position will be dragged onto the map (i.e., Google Maps) showing the designed parking points. Thus, after verification of the user's position and indication, correspondingly the system measures and displays all the real positions of parking points, with having the information of vacant and nonvacant parking lots, over the map. Here, the user can be to select the near parking point which has vacant parking lots, so the request for parking (RFP) is submitted as a further step to make a reservation and then in case of confirmation that will be visualized into user login account.

3.3.5. Smart Payment. Like other smart features available in the SVPS system, the system also provides an efficient and user-convenient method to manage the parking payments; by employing this smart payment method, the user is not restricted to make payment, at the instance, after parking usage or checking out the parking point. As mentioned above, the system has been fully functional on per hour basis; for example, in case the vehicle only spent 30 minutes or more, but less than 1 hour, then the system still computes the parking payment on an hourly basis. However, there will be a margin of almost 10 minutes, after spending an hour, using the vehicle to check out the occupied parking lot. Therefore, after exiting from parking, the total payment will be calculated by the system based on the time the vehicle occupied the parking lot, and the total amount will display inside the user's account. Thus, the user could view the total parking payment or the parking payment history, according to the usage time of the login.

Each time the user uses the system' parking service, the equivalent parking fee (or amount) is calculated and aggregated in the total which should be paid by the user. The user has options to make the payment by the end of the following month, through using various payment methods including the direct account deposit and cheque methods. Further, the users who are permitted to make payments via credit/debit card have to register prior to using the SVPS system. Thereby, the desired total parking payment will be automatically paid at the end of the month or according to the due date. However, these payment methods are now not very new, as an inherent property usually employed by the advanced banking systems, over the world.

3.4. User Application Layer. At the application layer or the abstraction layer, typically all the main employing protocols, interfaces, and system' services are resided and defined to be used by the end-users perspective. Therefore, the SVPS system users could get access to the system available services (or resources), using various smart devices, facilitating through the user application layer.

\section{Smart Parking Design and Setup}

For instance, the SVPS system's design is comprised of 20 parking points $(\mathrm{PP})_{i}$, that is, $(\mathrm{PP})_{i}=\left\{(\mathrm{PP})_{1},(\mathrm{PP})_{2},(\mathrm{PP})_{3}\right.$, $\left.\ldots,(\mathrm{PP})_{n}\right\}$, where $n$ represents the $n$th parking point, locating at various crowded main parts (or places) of metropolitan city (i.e., Seoul city of South Korea) where most of the popular companies, shopping malls, and other daily working places are usually situated. Each parking point, PP, in total of $(\mathrm{PP})_{(i \leq n)}=20$, has occupied distinct number of parking lots $(\mathrm{PL})_{e}$, that is, $(\mathrm{PL})_{e}=\left\{(\mathrm{PL})_{1},(\mathrm{PL})_{2},(\mathrm{PL})_{3}, \ldots,(\mathrm{PL})_{k}\right\}$ and $1 \leq$ $k \leq 150$. Thus, the number of parking lots $(\mathrm{PL})_{e}$ in each parking point $\mathrm{PP} \in(\mathrm{PP})_{i}$ is depending on the total area size; for example, the standard maximum area for each designed parking point is not fixed but might surrounded in area of several acres. Similarly, the size (width $*$ length) of each parking slot PL is also not defined in a fix size and varies to the vehicle size, but the standard size used is approximately 8 feet by $16 \mathrm{feet}$, that is, $8 \mathrm{ft}$ wide and $16 \mathrm{ft}$ long. However, this study is not under considerations of that to encompass multiple levels parking; the parking points $(\mathrm{PP})_{i}$ are designed in open area but covered with the boundary walls. However, each parking point (PP) has different area occupied for vehicle parking with distinct number of parking lots $(\mathrm{PL})_{e}$, such that $e \leq k=150$. 
TABLE 1: Designed parking points and mapping description.

\begin{tabular}{|c|c|c|c|c|c|c|c|}
\hline Number & P entitled & Number of gates & Direction & Number of PL & PL size & Number of nodes (slave; master; anchor) & Local mapping \\
\hline (1) & PP01 & \multirow{20}{*}{2} & Northeast & 120 & \multirow{20}{*}{$8 * 16$} & $(124 ; 13$; var $)$ & \multirow{20}{*}{$\begin{array}{c}\text { Guidance map } \\
\text { available }\end{array}$} \\
\hline$(2)$ & PP02 & & Northeast & 120 & & $(124 ; 13 ;$ var $)$ & \\
\hline (3) & PP03 & & Northeast & 110 & & $(114 ; 12 ;$ var $)$ & \\
\hline (4) & PP04 & & Northeast & 90 & & $(94 ; 10 ;$ var $)$ & \\
\hline (5) & PP05 & & Southeast & 80 & & $(84 ; 09 ;$ var $)$ & \\
\hline (6) & PP06 & & Southeast & 90 & & $(94 ; 10 ;$ var $)$ & \\
\hline (7) & PP07 & & Southeast & 90 & & $(94 ; 10 ;$ var $)$ & \\
\hline (8) & PP08 & & Southeast & 130 & & $(134 ; 14 ;$ var $)$ & \\
\hline (9) & PP09 & & Southeast & 150 & & $(154 ; 16 ;$ var $)$ & \\
\hline (10) & PP10 & & Southeast & 140 & & $(144 ; 15 ;$ var $)$ & \\
\hline (11) & PP11 & & South & 90 & & $(94 ; 10 ;$ var $)$ & \\
\hline (12) & PP12 & & Southwest & 90 & & $(94 ; 10 ;$ var $)$ & \\
\hline (13) & PP13 & & Southwest & 140 & & $(144 ; 15 ;$ var $)$ & \\
\hline (14) & PP14 & & Southwest & 150 & & $(154 ; 16 ;$ var $)$ & \\
\hline (15) & PP15 & & Southwest & 140 & & $(144 ; 15 ;$ var $)$ & \\
\hline (16) & PP16 & & Northwest & 150 & & $(154 ; 16$; var $)$ & \\
\hline (17) & PP17 & & Northwest & 145 & & $(149 ; 16$; var $)$ & \\
\hline (18) & PP18 & & Northwest & 115 & & (119; 13; var) & \\
\hline (19) & PP19 & & Northwest & 125 & & $(129 ; 14 ;$ var $)$ & \\
\hline (20) & PP20 & & Northwest & 80 & & (84; 09; var) & \\
\hline
\end{tabular}

$$
\begin{aligned}
& \operatorname{Sy}_{\text {SVPS }} \ni\left[(\mathrm{PP})_{(i, i \leq n)}\right], \\
& \text { where } i \text { is a integer and } i \leq n=20 \\
& \Longrightarrow(\mathrm{PL})_{e}=\left[(\mathrm{PL})_{(e, e \leq k)}\right], \\
& \text { where } e \text { is a integer and } e \leq k=150 \\
& f(X)=\left[(\mathrm{PL})_{(e, e \leq k)}\right] \in \sum_{t=1}^{l}(x)_{t}, \\
& \quad \Longrightarrow f(X) \cdot(\mathrm{PL})_{e} .
\end{aligned}
$$

SVPS system, $\mathrm{Sy}_{\mathrm{SVPS}}$, encompasses distinct parking lots $(\mathrm{PL})_{e}$ in designed parking points $(\mathrm{PP})_{i}$, but each parking point $\mathrm{PP}$ has occupied $(\mathrm{PL})_{e} \leq k$, the total number of parking lots $(\mathrm{PL})_{e}$ at each parking point PP is computed in (3) and (4). $f(X) \cdot(\mathrm{PL})_{e}$, where $f(X)$ aggregates the value of $(x)_{t} \leq$ $l$ upon each computation from $(\mathrm{PL})_{e} \leq k$, depicted in Table 1 including overall required parameters detail. Further, a function is defined $f(Y)=f(X) \cdot(\mathrm{PL})_{e}$ in order to compute the number of nonvacant $\mathrm{NV}$, that is, $(e, t)=\emptyset$, vacant $V$, that is, $(e, t) \leq(k, l)$, and parking lots $(\mathrm{PL})_{e}$ by internal system computations.

$$
f(Y)= \begin{cases}f(X) \cdot(\mathrm{PL})_{e}, & (e, t) \leq(k, l) \\ f(X) \cdot(\mathrm{PL})_{e}, & (e, t)=\emptyset\end{cases}
$$

The information in Table 1 shows the general-purpose analyzed information used during designing phase of parking points, as part of SVPS system. As shown, for each parking point $\mathrm{PP}$, number of parking lots $(\mathrm{PL})_{e}$ and the nodes (or sensor nodes) employed to carry the information are listed, including other important details. However, the number of anchor nodes employed in network setup of each parking point is variable (Var) in size, and also the direction mentioned for each parking point PP is measured as an closed estimation (not exact estimation).

Moreover, in Figure 3, a graphical representation is made to show the number of parking point $(\mathrm{PP})_{i}$ distribution at the various selective locations and with the appropriate directions, over the SVPS-Map positions.

4.1. Network Configuration. In SVPS system, each parking point $\mathrm{PP} \in(\mathrm{PP})_{i}$ is completely designed and networked under the considerations of efficient computing paradigms; thus, it is assumed that all the slave-sensor nodes (SS nodes) are installed at the optimal positions where they could communicate with the master-sensor node (MS node), and further, to local system controller (LSC). In parking points $(\mathrm{PP})_{i}$, as mentioned above, the available parking lots $(\mathrm{PL})_{e}$ sizes are not fixed but the standard size used is about 8 feet by 16 feet (width $*$ length). Each parking lot $(\mathrm{PL})$, in $(\mathrm{PP})_{i}$, is equipped with slave-sensor node (SS node) or RFID reader installed at the ground position with the protection of solid shield; the ground position is exactly at the intermediate middle position of the PL entrance. Therefore, the registered system's vehicle with the embedded RFID tag should communicate with the SS node installed, using RFID air-interface protocol.

In Figure 4, the identification of vehicle at the parking lot is made through RFID tag that fixed the vehicle's number plate, under the vehicle's front-bonnet; thereby the vehicle 


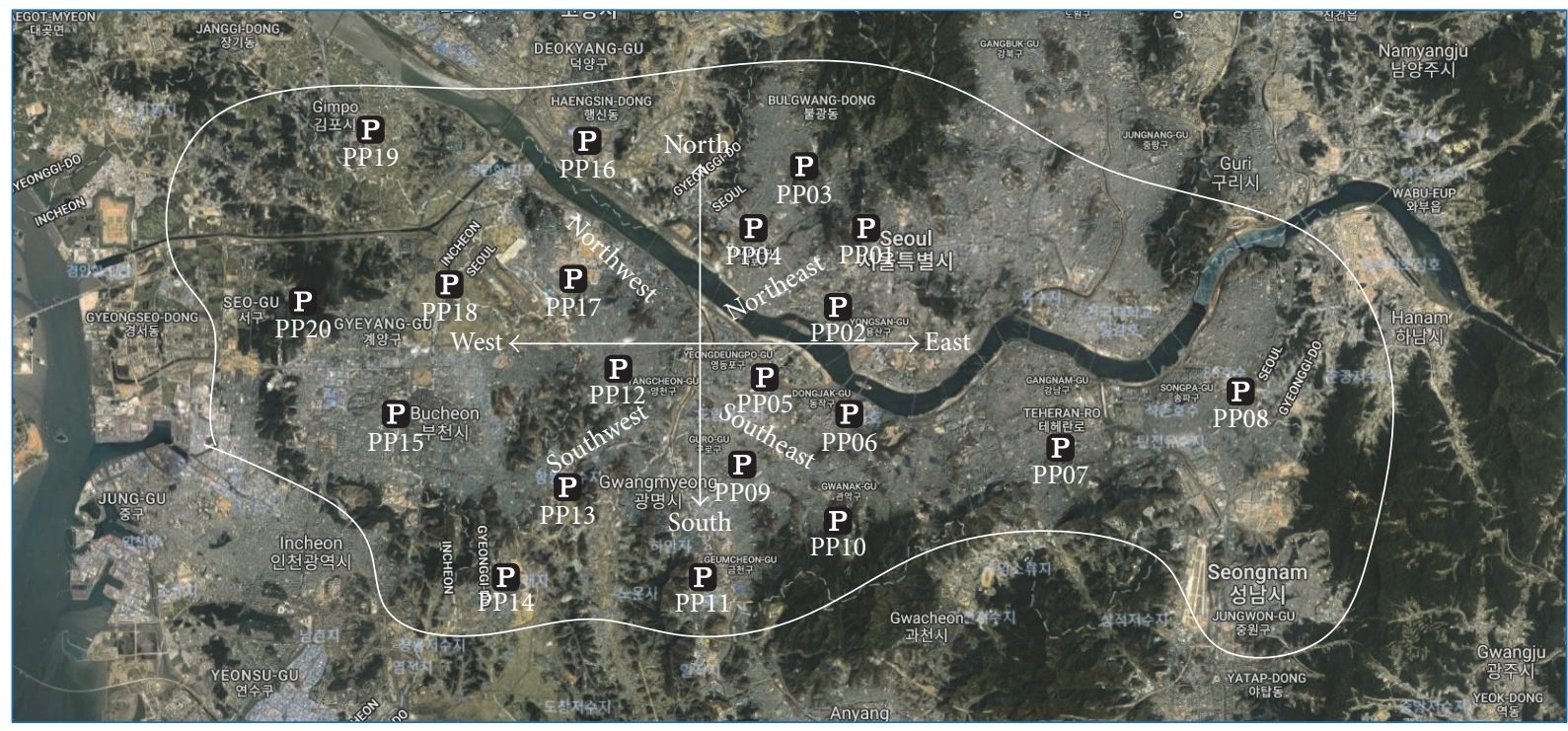

FIGURE 3: Graphically distribution of parking points.
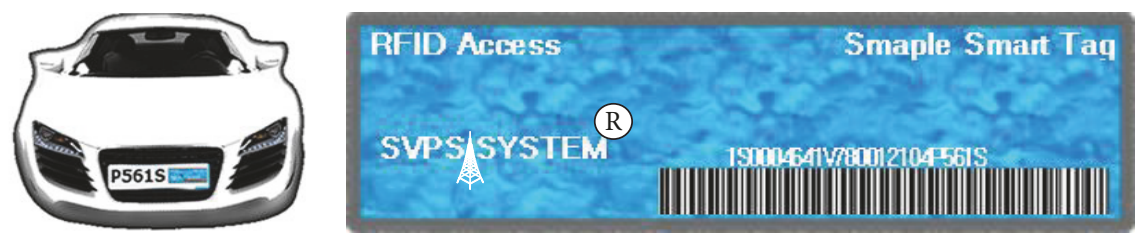

FIGURE 4: Smart parking RFID tag and installation.

gets reliable access while entering into the parking lot (PL) assigned. The installation or the fixing process of RFID tag on each vehicle, after registration, is twofold:

(1) In general, the vehicle's number plate is important for the verification purposes in daily life, thus the fixing of RFID tag on vehicle plate will be very useful to get that vehicle identification and verification, at the time vehicle enters into parking point, $\mathrm{PP}$.

(2) As mentioned, each parking lot (PL) is equipped with slave-sensor node installed at the ground position; thus information will be reliably and easily accessible upon vehicle entry at the allocated PL. Through the RFID technology, unlike a barcode, there is not a requirement that RFID tag must be aligned or placed in the line of sight, to the RFID reader installed at each parking lot $(\mathrm{PL})_{e}$.

For instance, in Figure 5, the number of parking lots $(\mathrm{PL})_{e}$ is variable sizes in each parking point (PP), but the maximum ten parking lots $(\mathrm{PL})_{(e \leq k=10)}$, that is, $\operatorname{Max}\left\{1 \leq(\mathrm{PS})_{e} \leq\right.$ $10\}$, are assigned to each master-sensor node (MS node), connecting with the wired connection and communication made through the $\mathrm{I}^{2} \mathrm{C}$ protocol. RFID technology takes the benefits from WSN technology to increase the converge area, because mainly the RFID systems have been deployed to access the object or the node has RFID tag embedded on, within the short range of about 2 meters (or more). The WSN system efficiently extends the wireless coverage of RFID devices to be reachable to the controller, for example, while transmitting information from sensor nodes to the local system controller in our case. In short, the master-sensor nodes (MS nodes) are directly configured and connected with the slave-sensor nodes installed in parking lots, performing the information read/write functions, and are responsible for transmitting the observed information, through anchor nodes, to local LSC residing in each parking point (PP). Further, MS nodes are responsible for performing controlling operations, through connected slave-sensor nodes (SS nodes), instructed from the CC system. In each parking point, the employed MS nodes are connecting with each other, so each MS node has fully awareness about its neighboring MS node. For controlling and monitoring, the CC system is always responsible; the used RFID technology is flexible and programmable in its usage, according to the commands and instructions. Therefore, the networked system is efficiently regulated through the commands of CC system.

For example, in Table 1, the parking point $(\mathrm{PP})_{(i=12)}$, or PP12, occupied 90 parking lots $(\mathrm{PL})_{(e=90)}$. Thus processing through CC system's database, as a result, few parking lots $(\mathrm{PL})_{e}$ are vacant $(\mathrm{V})$, that is, $\mathrm{V} \in(\mathrm{PL})_{e}$, such as SVPS-L006, SVPS-L008, SVPS-L010, SVPS-L44, SVPS-L55, and SVPSL74, and further suppose that rest of them are nonvacant $(\mathrm{NV})$, that is, $\mathrm{NV} \in(\mathrm{PL})_{e}$. From CC system, suppose that, 

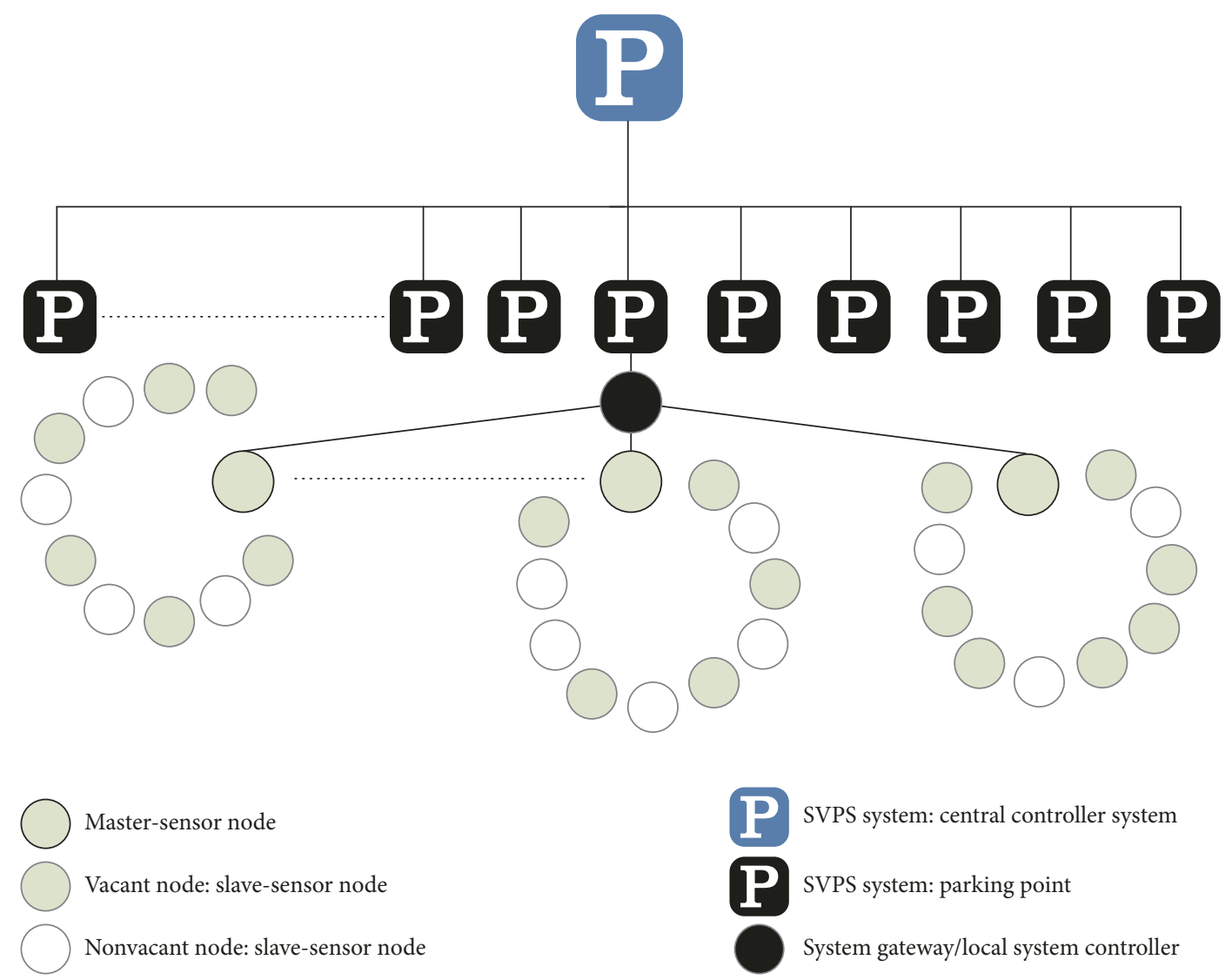

SVPS system: central controller system

D SVPS system: parking point

System gateway/local system controller

FIGURE 5: SVPS system: sensor nodes connectivity.

based on the reservation records, the following information is collected:

(1) The six vehicles having vehicle registration IDs $U_{\text {Reg }}, U_{\text {Reg }} \in \mathrm{Sy}_{U} \in \mathrm{Sy}_{\mathrm{SVPS}}$, such as P561S, T782E, TT89D, TS90P, PK61L, and PK61L.

(2) Registered tag numbers $U_{\text {Tag }}, U_{\text {Tag }} \in U_{\text {Reg }} \epsilon$ $\mathrm{Sy}_{U} \in \mathrm{Sy}_{\mathrm{SVPS}}$, like IS0004641V780012104P561S, IS0205841P707012104S535S, and so on, illustrated in Figure 5.

(3) Registered users IDs, $U_{\mathrm{ID}}$, $\left(U_{\mathrm{Tag}}, U_{\mathrm{Reg})} \in U_{\mathrm{ID}} \in \mathrm{Sy}_{U} \in\right.$ Sy ${ }_{\text {SVPS }}$, like Tommy-IS0004641V780012104P561S, Alice-IS0205841P707012104S535S, and so on.

(4) Allocated parking lots $(\mathrm{PL})_{e}$, that is, $\mathrm{V} \in(\mathrm{PL})_{e}$, such as PP12-PL065, PP01-PL061, PP01-PL025, PP01PL022, PP01-PL001, and PP01-PL006.

For each SVPS system's user $(U)$ or $S_{U} \in S_{S V P S}$, the above-mentioned detail is compulsory to be registered, a requirement for reservation. Therefore, the information analyzed above, accessing CC system, only those vehicles (e.g., vehicles P561S-PK61L) could get access at the entry gate and onto the designated parking lots (PP12-PL-065-PP01$\mathrm{PL}-006)$ in selective parking point $(\mathrm{PP})_{(i=12)}$. The installed slave-sensors nodes are smart and programmable and fully controlled under the commands given by the CC system. As a result, only that vehicle makes entry onto the selective parking lot of parking point commanded by the CC system. In case, the vehicle enters into the correct parking point, through the reading of sensors installed at the main barrier, mistakenly, the same vehicle will try to make parking onto the parking lot that is not designated for it. Then, upon identification, the installed slave-sensor node will not allow for parking and together generate the indication with red light, a sign that shows the unauthorized parking due to the vehicle identification failure.

For security and ensuring of each registered user (or registered vehicle) under SVPS system, in Figure 6, the main entrance (of parking point) is equipped with two sensors, only at the main entrance. However, the exit gate or exit barrier has one sensor installed to acquire the reading during exit. Thus, at the entrance to the selective parking point (that authorized by the SVPS system), two sensors installed will be active for the vehicle's identification, operating through the follwoing:

(1) The first sensor is installed at the parking entry gate; similar position as parking lot has, at the center of entrance, one step backward of parking boom barrier.

(2) At the same time and the place exactly at the left side, another sensor device is fixed and activated on the panel, which inclusively makes identification of each vehicle at entry point through only with smart RFID card provided by the SVPS system. 

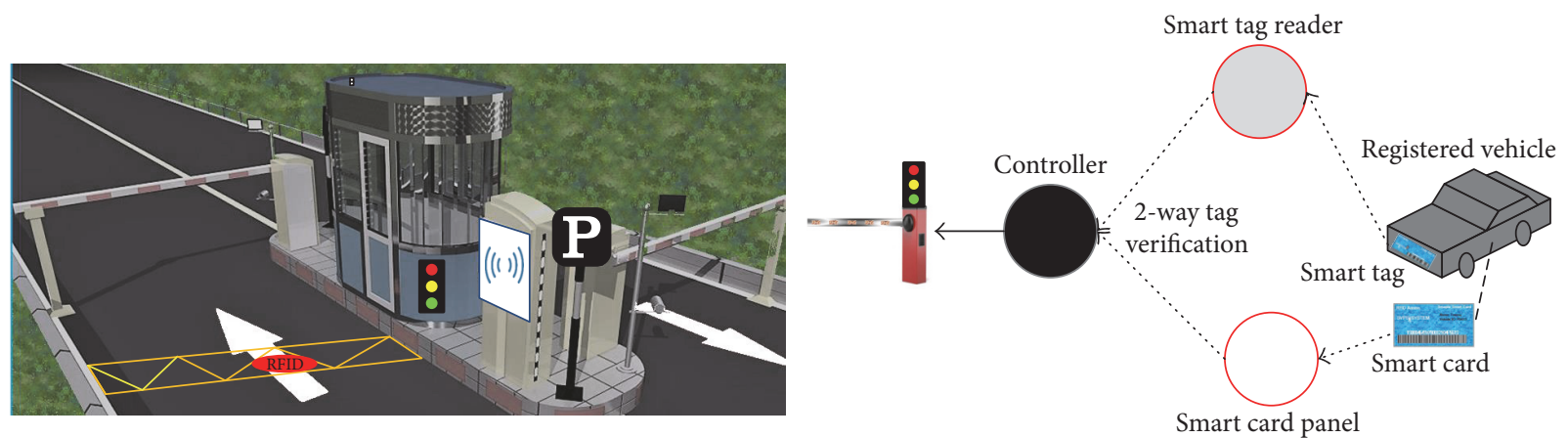

Figure 6: Nodes identification and verification.

\section{System Implementation}

For parking reservation or request for parking (RFP), users are allowed to access the service online through accessing the SVPS system which keeps the record, in real time, of both nonvacant $\mathrm{NV}$ and vacant $V$ parking lots from the parking points, such that $f(Y)=\left\{f(X) \cdot(\mathrm{PL})_{e} \mid \mathrm{NV} ; \mathrm{V}\right\} \in$ $(\mathrm{PP})_{i} \in \mathrm{Sy}_{\mathrm{SVPS}}$; for that, the information is entirely computed from master-slave-sensor nodes (M2S nodes). Therefore, the system users $S y_{U}$ (i.e., $S y_{U} \in S y_{\text {SvPS }}$ ) are ensured for their reserved parking lots $(\mathrm{PL})_{e}$ before arriving at the selective distinct parking points $(\mathrm{PP})_{i}$. However, some time limitations, but very flexible, are also mentioned and restricted from the SVPS system which enables the session for the user to be reached and used his/her reserved parking lot; further, while the session expires, the SVPS dynamically allocates that parking lot (e.g., the occupied PL) to other users waiting inside system reservation queue $\left(S y_{R Q}\right)$. The SVPS system used an efficient method to manage and regulated all the reservations pending in the system reservation queue; while it is vacant, the parking lots are assigned to the users who are waiting inside the queue; however the priority scheme should be a main consideration.

SVPS system online is only available for the users that are registered under the system, known as authorized users of the system; other entities are not allowed at all or considered as unauthorized entities. The SVPS system web application is designed, in mind, to be easily usable by the system's users. While successfully logging in to the system online, the user will have access to the various services available. Therefore, the user could access all available services enabled for the desired parking searching and reservation purposes and view the corresponding parking payment details inside the account. To be convenient, the interactive inclusive SVPS-Map is available where all the parking points (i.e., 20 parking points) are located, being useful for the users who are planning to do searching for the closer parking points according to the positions where they are. Thus, based on the user selection for parking point, the user is further allowed to see all the available parking lots, for example, nonvacant $(\mathrm{NV})$ and vacant $(\mathrm{V})$. However, from the system regulations, each user is limited to do reserve the parking lot only once at a time, without any time constraints; but the user could allow reserving another parking lot in distinct parking point in case the user lefts or checks out from the current parking lot that he/she occupied. For payment, the system is designed to manage the total payment of parking service, the user parking utilization is computed on an hourly basis and has opted to make payment by several available reliable methods.

5.1. Smart Access and Reservation. During online SVPS system access, suppose a registered user $U$ has valid entity such that $U \in$ Sy $_{\text {SVPS }}$. Thus, upon entering the valid user identification ID and password PW, that is, $U($ ID; PW) $\epsilon$ Sy ${ }_{\text {SVPS }}$, the login credentials are authenticated and then user $U$ is permitted to enter into the system. As a newly entry into the system, the user is allowed to use the overall system' services available inside his/her account, for example, especially the parking searching and reservation service. For that, a request for parking (RFP), the system efficiently displays all the available parking points $(\mathrm{PP})_{i}$, over the geographical positions designated, on the SVPS-Map, illustrated in Figure 3. Over the SVPS-Map, all the parking points are located a distance far from each other, about the distance of kilometers; the parking point distribution is appropriated for the users who could do the parking reservations over the various locations where they are looking for parking.

$$
\begin{aligned}
U & \in \text { Sy }_{U} \in \text { Sy }_{\text {SVPS }} \\
U(\mathrm{ID} ; \mathrm{PW}) & \in \text { Sy }_{\text {SVPS }} \\
& \Longrightarrow f(U)= \begin{cases}0, & U \in \text { Sy }_{\text {SVPS }} \\
\Phi, & U \notin \text { Sy }_{\text {SVPS }}\end{cases}
\end{aligned}
$$

$f(U)$ is a function defined to authenticate the user $U(I D ; P W)$, at the time of login to SVPS system online. In case system authenticates the user $U$, that is, $U(I D ; P W)=0$, then user is allowed to use the service RFP for any parking point $\mathrm{PP} \in(\mathrm{PP})_{i}$,

$$
\exists f(U)=0 \Longrightarrow\left[(\mathrm{PP})_{(i, i \leq n)}\right], \quad i=[1,2,3, \ldots, n] .
$$

Thus, all the available parking points $(\mathrm{PP})_{i}$ will be displayed to the user, prevalent over various positions onto the SVPSMap; here user has two options to do searching for the closer parking points $(\mathrm{PP})_{i}$ and then do a request for parking, which are as follows: 
(1) Selective smart parking (SSP): user inputs the specific location as a source location and then searching the closer parking point through SVPS-Map displays all the parking points that are nearest to the selective location of the user. However, the system shows the parking points starting from the closer once, computing the distance from the selective location to each parking points $(\mathrm{PP})_{i}$. For example, in Figure 7, the user inputs a location as his/her target location and requires getting the closest parking points. For that, the system computes the distance between the target position of user and all the available parking points' positions and thus displays the closer parking points visualized in red color, in converge of red cell, in number order of PP18, PP17, PP12, and PP15.

(2) Smart positional parking (SPP): for this, a request for parking (RFP), the system using SVPS-Map gets automatically the current location of the user and then according to target location input, a number of parking points will be displayed starting from the closest once. For example, in Figure 8, the system gets a current position of the vehicle or a user position and computes the distance with all the available parking points' positions. As a result, the system displays the closer parking points, visualized in red color, in converge of the red cell, with the numbers 01 and 02 . Thus, it is concluded that the PP01 has a less distance compared with PP02 within converge of the red cell.

In both cases, the input target location and its distance are computed with all the available parking points and according to the computed results the closer parking points one after another will be identified based on the distance difference with and displayed to the user. However, the total distance between all the available parking points is known by the system, while in design and setup phase of SVPS system.

Mathematical Formulation. Suppose that a function $f(d)$ computes the distance $d$ between each of available parking points $(\mathrm{PP})_{i}$ such that Sy SvPS $_{\text {: }} f(d)=d\left[(\mathrm{PP})_{i}\right]_{(a b)}$, where $a$ and $b$ are the distance coordinates of each parking point $\mathrm{PP} \in(\mathrm{PP})_{i}$. As, at instance, the number of parking points $(\mathrm{PP})_{i}$ are limited to integer $n$. Thus, the function $f(d)$ computes the distance between parking points $(\mathrm{PP})_{(i, i \leq n)}$ followed by the value $\mathcal{S}$.

$$
\begin{gathered}
\mathcal{S}=\left\{(\mathrm{PP})_{1},(\mathrm{PP})_{2},(\mathrm{PP})_{3}, \ldots,(\mathrm{PP})_{n}\right\}=(\mathrm{PP})_{(i, i \leq n)} \Longrightarrow \\
f(d)=d[\mathcal{S}]_{(a b)}=d\left[\begin{array}{c}
\left\{(\mathrm{PP})_{1},(\mathrm{PP})_{2}\right\} ;\left\{(\mathrm{PP})_{1},(\mathrm{PP})_{3}\right\} ;\left\{(\mathrm{PP})_{1},(\mathrm{PP})_{4}\right\} ; \ldots ;\left\{(\mathrm{PP})_{1},(\mathrm{PP})_{n}\right\} \\
\left\{(\mathrm{PP})_{2},(\mathrm{PP})_{3}\right\} ;\left\{(\mathrm{PP})_{2},(\mathrm{PP})_{4}\right\} ;\left\{(\mathrm{PP})_{2},(\mathrm{PP})_{5}\right\} ; \ldots ;\left\{(\mathrm{PP})_{2},(\mathrm{PP})_{n}\right\} \\
\left\{(\mathrm{PP})_{3},(\mathrm{PP})_{4}\right\} ;\left\{(\mathrm{PP})_{3},(\mathrm{PP})_{5}\right\} ;\left\{(\mathrm{PP})_{3},(\mathrm{PP})_{6}\right\} ; \ldots ;\left\{(\mathrm{PP})_{3},(\mathrm{PP})_{n}\right\} \\
\left\{(\mathrm{PP})_{4},(\mathrm{PP})_{5}\right\} ;\left\{(\mathrm{PP})_{4},(\mathrm{PP})_{6}\right\} ;\left\{(\mathrm{PP})_{4},(\mathrm{PP})_{7}\right\} ; \ldots ;\left\{(\mathrm{PP})_{4},(\mathrm{PP})_{n}\right\} \\
\left\{(\mathrm{PP})_{5},(\mathrm{PP})_{6}\right\} ;\left\{(\mathrm{PP})_{5},(\mathrm{PP})_{7}\right\} ;\left\{(\mathrm{PP})_{5},(\mathrm{PP})_{8}\right\} ; \ldots ;\left\{(\mathrm{PP})_{5},(\mathrm{PP})_{n}\right\} \\
\left\{(\mathrm{PP})_{6},(\mathrm{PP})_{7}\right\} ;\left\{(\mathrm{PP})_{6},(\mathrm{PP})_{8}\right\} ;\left\{(\mathrm{PP})_{6},(\mathrm{PP})_{9}\right\} ; \ldots ;\left\{(\mathrm{PP})_{6},(\mathrm{PP})_{n}\right\} \\
\vdots \\
\vdots \\
\vdots
\end{array}\right. \\
\left\{(\mathrm{PP})_{n-1},(\mathrm{PP})_{n}\right\}
\end{gathered}
$$

For example, the distance $d$ between $(\mathrm{PP})_{1}$ and $(\mathrm{PP})_{2}$ is computed as

$$
\begin{aligned}
& d\left[(\mathrm{PP})_{1},(\mathrm{PP})_{2}\right]_{(a b)} \\
& =\sqrt{\left[(\mathrm{PP})_{1}(a)-(\mathrm{PP})_{1}(b)\right]^{2}+\left[(P P)_{2}(a)-(\mathrm{PP})_{2}(b)\right]^{2}} .
\end{aligned}
$$

Further, assume that $L_{1}$ and $L_{2}$ are the target location and the current location of registered user $U$ input, then the system is mapping the input values $L_{1} \mapsto d[\mathcal{S}]_{(a b)}$ and $L_{2} \mapsto d[\mathcal{S}]_{(a b)}$ and resulted in the closer parking points $(\mathrm{PP})_{i}$ visualized in Figure 9.

For the user convenient and based on the location inputs, in general, the system executes the user request and then displays the available parking points $(\mathrm{PP})_{i}$ in order starting from the closest to that user's input location. However, in Figures 7 and 8, the system only displayed the closer parking points, that is, PP18, PP17, PP12, and PP15 and PP01 and PP02, with an indication of available or vacant parking lots $(\mathrm{PL})_{e}$. More precisely, in Figure 9, the indication symbol in red and black colors shows the number of vacant $\mathrm{V}$ parking lots $(\mathrm{PL})_{e}$, such as 10 in PP01, 23 in PP02, 44 in PP18, 31 in PP17, and 52 in PP15; however, in case the parking lots $(\mathrm{PL})_{e}$ are not vacant $\mathrm{NV}$, then they are displayed with zero, for example, in case of PP12.

In Figure 9, using the cases of Figures 7 and 8, the system computed a number of vacant $\mathrm{V}$ parking lots $(\mathrm{PL})_{e}$ indicated individually for the selective parking point $(\mathrm{PP})_{i}$, but not 


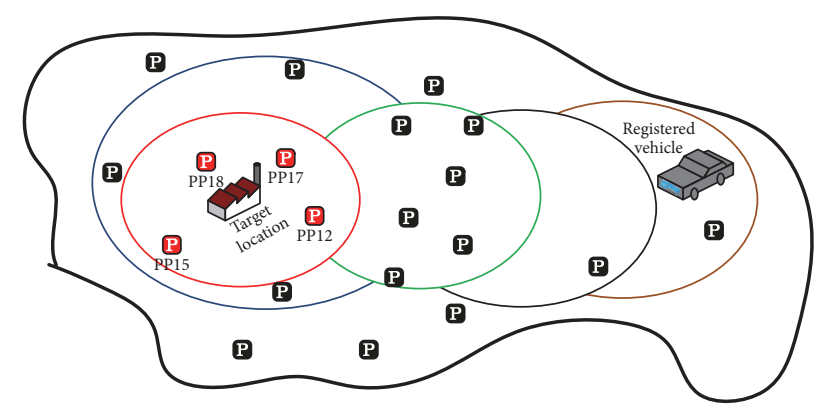

FIGURE 7: Selective smart parking using SVPS-Map.

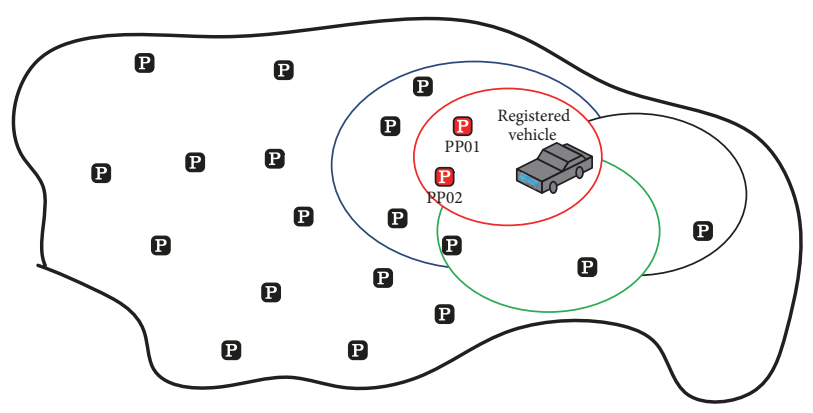

FIGURE 8: Smart positional parking using SVPS-Map.

for all. Therefore, during request for parking, users save time by knowing about the vacant (or nonvacant) parking lots in advance before they go to do parking reservations. The parking reservation became more straightforward to the users, as they already know the available parking points and the vacant parking lots, to submit the request for parking. The SVPS system employed a general-purpose strategy to design and visualize the number parking lots in parking points, which is useful at the time of parking reservations. In Figure 10, for example, PP01 occupied 120 parking lots in consecutive order and is displayed in the form of fixed size blocks; the vacant parking lots are represented in white color and at the same time, the gray color represented nonvacant parking lots. Thus, through request for parking, user has an option to select any of parking lots among the vacant parking lots. By selecting the specific parking lot, for example, parking lot number PP01-PL010 shown in white color, further system processes a request for parking and allocates it for the user who requested. At the same time, the allocated parking lot PP01-PL010 information is updated in the database, and the corresponding master-slave-sensor nodes are triggered to allow access to the authorized vehicle to do parking (through reading of RFID tag information). Following the user request for parking, that is, fixed time parking and timed parking, the session for parking (to utilize the allocated parking lot) is added, and the master-slave-sensor nodes are very known or have information that allows the vehicle to do parking according to the session mentioned and exit while time finished. However, the user has option to extend the existing declared session of parking using SVPS system online; otherwise, the system regulates the parking timing on an hourly basis, for example, using any time parking feature.

For information acquisition, in short, while user request for parking is confirmed and reserved with the allocation of the parking lot, the system actives the sensor nodes with the user (or vehicle) registered ID using write function; therefore only that user could do the parking onto the parking lot allocated. All the operations, such as read and write operations, fully instruct, operate, and are controlled by the central controller system (CC system), and the corresponding computed information is always simultaneously updated into the system database or SVPS-DB. In Figure 11, SVPS-DB designed (or created) tables show the overall information keep while each time the system gets reading from masterslave-sensor nodes (i.e., slave-sensor nodes) installed at the parking point $\mathrm{PP} \in(\mathrm{PP})_{i}$. Further, some of important operations performed and managed in SVPS-DB are following:

(1) For each parking point PP $\in(\mathrm{PP})_{i}$, SVPS-DB has fixed number of tables for keeping the information up to date from the master-slave-sensor nodes. However, it depends directly on the number of parking lots $(\mathrm{PL})_{e}$ being installed with the sensor nodes in each parking point. For example, suppose that, in parking point 03 or PP03, total number of parking lots are 114 installed independently with the sensor nodes; therefore for information acquisition, the starting 110 parking lots are directly connected and managed through 11 master-sensor nodes, that is, 10 slavenodes/1 master-sensor node, and the remaining 4 parking lots out of 114 are through 12th mastersensor node. This means overall 114 slave-sensor nodes are managed through 12 master-sensor nodes; more details are depicted in Table 1 showing the information of each parking point installed with the fixed number of master-slave-sensor nodes. Thus, in the SVPS-DB, the system maintains 12 tables to keep the information individually from 12 master-sensor nodes designated to control the fixed number of slavesensor nodes installed to get the reading while vehicle checking in and checking out. However, information collected from master-slave-sensor nodes has also replicated in local system controller (LSC) setup in each parking point.

(2) Further, among other several tables, the system maintains two main tables in SVPS-DB: (1) parking status table and (2) parking for a request or parking reservation table. Parking status table keeps the record/information of parking lots, which are vacant and nonvacant based on the master-slave-sensor nodes reading information, from each parking point. Then, the collected information will be displayed to the users while searching for parking using SVPS system online. At the other side, parking for request table keeps the record of each reservation made by the users and the equivalent information that slavesensor nodes are fully activated only for registered vehicles having RFID tags. 


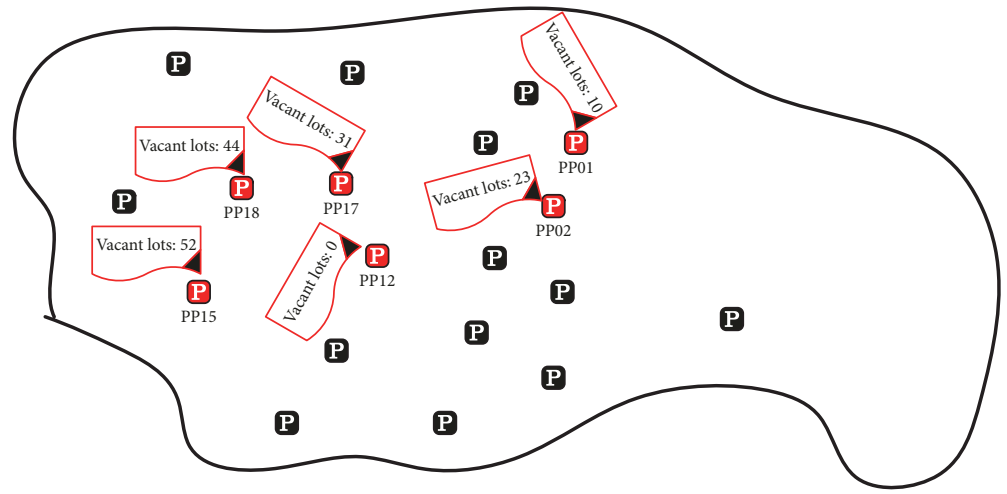

FIGURE 9: Computation of vacant/nonvacant parking lots using SVPS-Map.

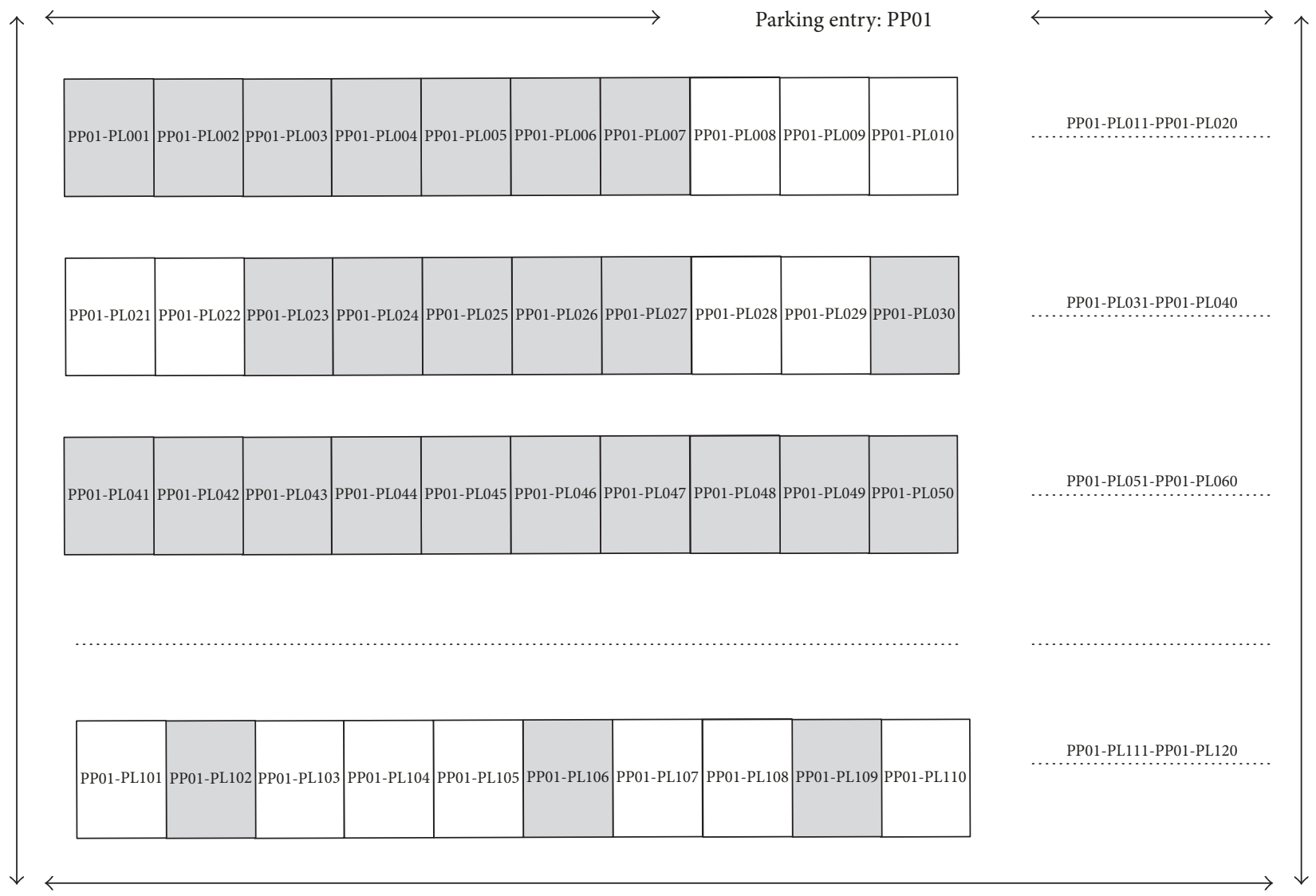

FIGURE 10: A design strategy for parking reservation.

\section{Results and Discussion}

At the time of user's login, the SVPS system authenticates the user entered ID and the password. Therefore, after authentication process, the user will be allowed access to use the available system's services; each time the user attempts to access the system services online, a session is activated for a fixed time (e.g., 9 minutes after first successful attempted of login) and can be further extendable. However, the current study or SVPS system is not designed under the consideration of efficient security paradigms in mind and only used the available general-purpose security mechanisms available inside operation system (i.e., firewall and commercial security tools) and conventional SSL and TLS solutions during the web searching. In early future, the study aims to design a fully secured security system that will fulfill all the requirements of security against the potential adversaries, while connecting with and managing the system online via Internet access.

For parking request, the overall system is efficient to manage the number of users in parallel. But in the situation, at same time, two or more users are targeting the same parking lot $\mathrm{PL} \in(\mathrm{PL})_{e}$ in parking point $\mathrm{PP} \in(\mathrm{PP})_{i}$, then the system 
TABLE 2: Write operation and computation.

\begin{tabular}{lccc}
\hline & & Function code, write operation: 1111 & Confirmation, bit set $=1$ \\
\hline 1 & System nodes & Execution & 1110 \\
2 & CC system to LSC & 1111 & 1100 \\
3 & LSC to MS node & 1110 & 1000 \\
4 & MS node to SS node & 1100 & 0000 \\
\hline
\end{tabular}

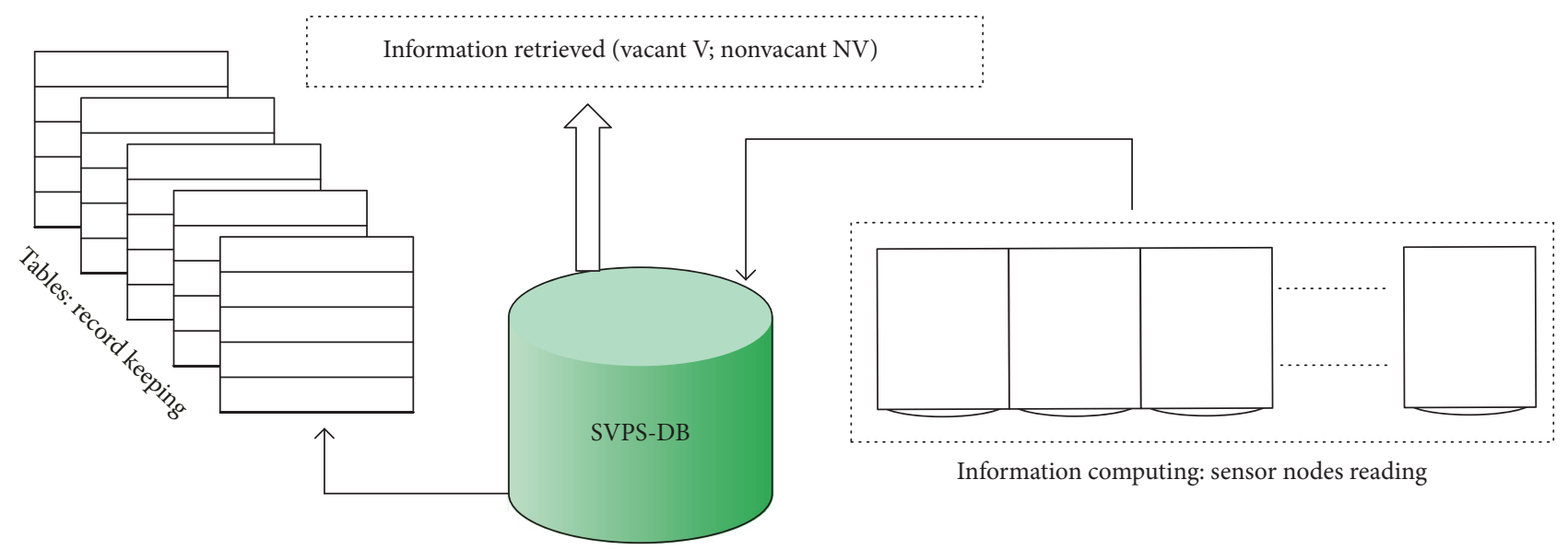

FIGURE 11: SVPS-DB: information computing and retrieving.

employs the scheduling algorithm, first-come, first-served (FCFS), and nonpreemptive approach, in order to manage the users. Suppose that, in Figure 11, three users input the request, request for parking, for the same parking lot PL01PL004 in parking point PP01, then the system computes the exact time (TR time of arrival) at which the first user among other has insert the request. Followed by the system, the time computed for user 02 is 02:12:55 (hour/minutes/seconds) which is less compared with the arrival times of user 01 and user 03 as 02:32:21 (hour/minutes/seconds) and 02:39:41 (hour/minutes/seconds). Therefore, a message or exception will be generated "the parking lot you are requesting is not available"; moreover, the other users are allowed any time to reserve the other available parking lots inside the same parking point. Here, in Figure 12, short IDs, such as user 01, user 02, and user 03, are used instead of long IDs defined by the system in Section 4.

The system is efficient in computing of information at each level, that is, sensor nodes to the local system controller to $\mathrm{CC}$ system and vice versa, of parking point $\mathrm{PP} \in(\mathrm{PP})_{i}$; therefore the users can get the overall required information in minimal time and in efficient manners. To compute and ensure the information at each level of computation, the general-purpose special functional codes are defined and employed by the system (each function code has 4-bit size) and are as follows: (1) for reading information, the code defined is 0000 , and (2) for writing information, the code defined is "1111." These are, for example, four-bit codes defined to perform the read and write functions from/to the master-slave-sensor nodes (M2S nodes). More precisely, the user-defined codes executing with the change of a bit at each level of computation during read and write operations. In the case of request for parking, the CC system performs the write operation to assign and activate the RFID reader or slave-senor node (SS node) installed at the parking lot with vehicle information having RFID tag fixed; thus upon the arrival of vehicle at the allocated parking lot the RFID tag information will read by the SS node. For that, CC system inputs a command "1111" for write operation; then the following execution will be performed at the each level of computation; that is, logically four levels are defined: (1) CC system to LSC, (2) LSC to MS node, (3) MS node to SS node, and (4) SS node. Thus, the write operation is performed as follows by these four levels: with the confirmation, bit set $=1$ changing at each level after successful execution.

In Table 2, the original code to write information is 1 , or write code $=1$, but the additional 3 bits or three 1's are used and exclusive or XOR operation is performed at each level. The bit set $=1$ is XOR with the least significant bit of "1111" at each level to get "0000" for confirmation. Therefore, at level 4 , the information is written using write code $=1$ (i.e., " 1000 ") and with the confirmation of "0000."

Similarly, in Table 3, CC system inputs a read code $=0$ to get information or reading from the SS node, for example, whether the parking lot is currently vacant or nonvacant, RFID tag information of the authorized vehicle and so on. For that, the additional three 0's are added to read code which becomes "0000" and for confirmation, a set bit $=0$ has been XOR each time with the least significant bit of " 0000 " to get finally the number of 1's or "1111" as confirmation observed 
TABLE 3: Read operation and computation.

\begin{tabular}{lccc}
\hline & \multicolumn{2}{c}{ Function code, reading operation: 0000} & Confirmation, bit set $=0$ \\
Levels & System nodes & 0000 & 0001 \\
1 & CC system to LSC & 0001 & 0011 \\
2 & LSC to MS node & 0011 & 0111 \\
3 & MS node to SS node & 0111 & 1111 \\
\hline
\end{tabular}

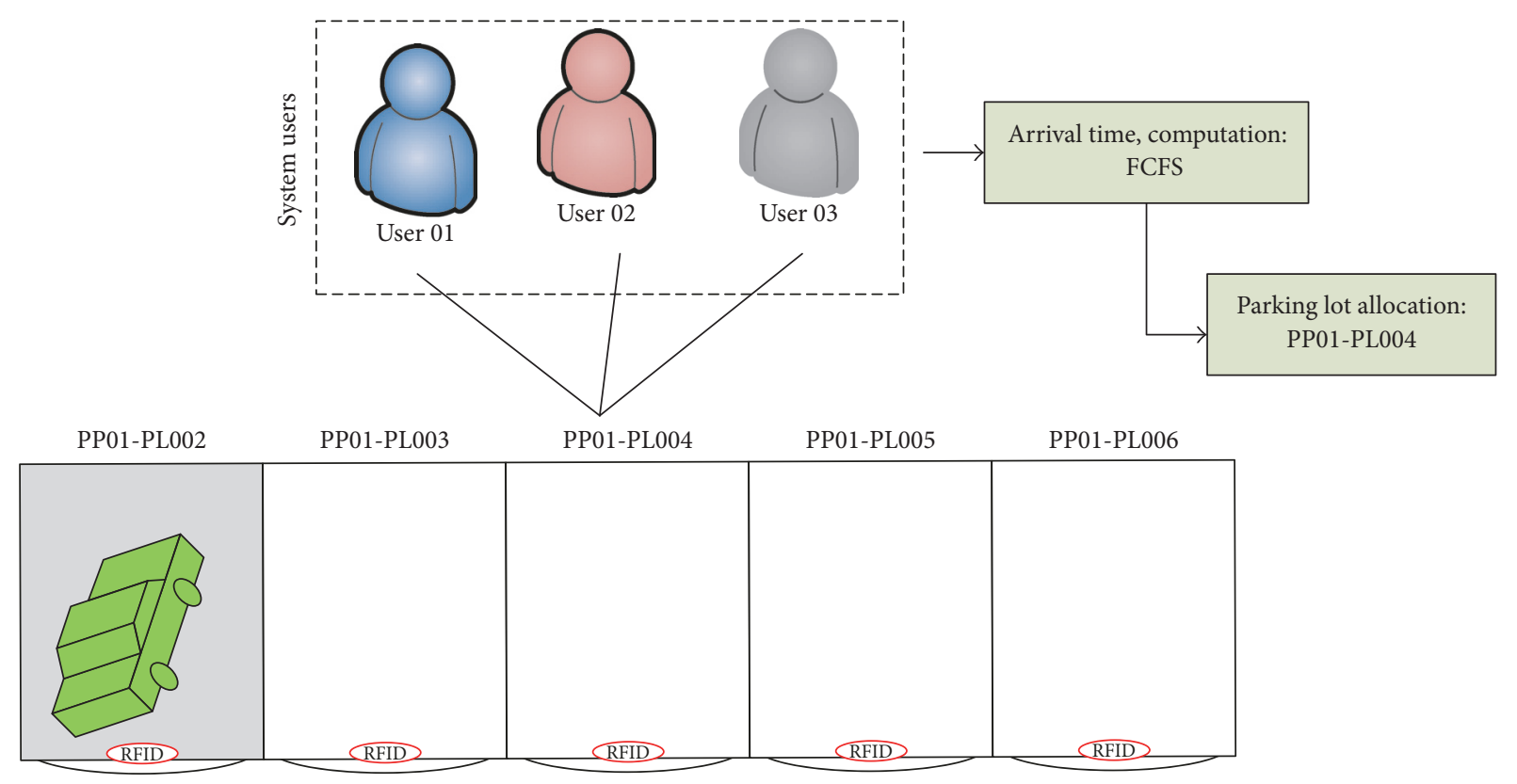

Figure 12: Parking allocation and management.

at each level. Further, the information being collected from RFID tag is intended to transmit back; according to command given by $\mathrm{CC}$ system, the sequence of the execution from nodes has been changed, as (1) RFID tag to SS node, (2) SS node to MS node, (3) MS node to LSC, and (4) LSC to CC system.

In Figure 13, the given information is the information transmitted from the parking point to the CC system. As mentioned, each parking point $\mathrm{PP} \in(\mathrm{PP})_{i}$ has been equipped or set up with various nodes to get instructions or commands and transmit reading information, from/to the CC system. For reading information, for example, the slave-sensor node gets reading from the RFID tag, and if the computed value is "0000" starting from the most significant bit or 0 bits which shows that the parking lot status is currently nonvacant, then confirmation set bit $=0$ is applied to get the value of " 0001 ." Continuously, at each level, set bit $=0$ is XOR in order to get the final confirmation at level 4 as "1111" and execution value "0111." Similarly, in Figure 14, slave-sensor node sets the value as "1111," meaning that the parking lot is currently vacant because the most significant bit is "1" and remaining three bits or 1's are added in order to get the confirmation at the next levels by applying set bit $=1$. Thus, at level $4, \mathrm{CC}$ system gets "1000" and with the confirmation "0000." Here, in both cases, whether parking lot is vacant or nonvacant, the computed values are the same as the values when CC system sends commands for write and read operations. Notably, each time command sent from CC system or sensor nodes replied the information back to CC system, the distinct identifications from them are also added along with the information. Thus, there is no conflict at all while command is transmitted or information is replied.

As a consequence, each piece of time information has been collected and transmitted back to the CC system and simultaneously kept storage into the SVPS-DB; therefore, the received information from the sensor nodes has been analyzed to acquire the number of parking lots whether being vacant or nonvacant individually from each parking point. While the slave-sensor node senses the vehicle tag information and upon verification, the vehicle will allow doing parking onto the parking lot, at the same time CC system gets the same information and updates the status of that parking lot as nonvacant. At the other side, upon the session completion assigned for the vehicle to utilize the parking lot, the vehicle has to be an exit from the parking lot; thus upon exit, the system updates the status of the parking lot as vacant for other users. However, in case the user wants to extend the parking session of same parking lot using SVPS system online, system keeps the parking status vacant till the vehicle exits. In Table 4 , the numerous vacant 


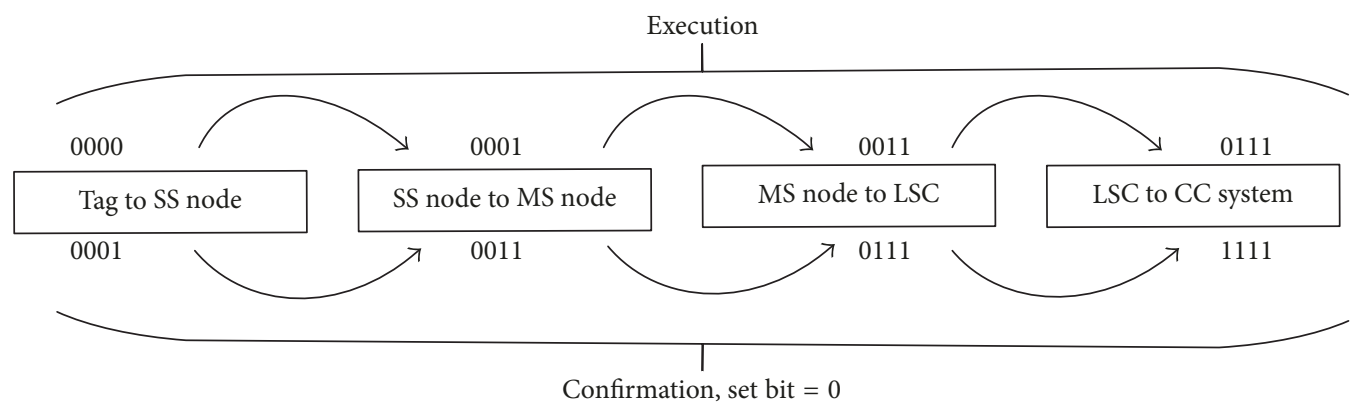

FIGURE 13: Nonvacant parking lot: reading information flow.

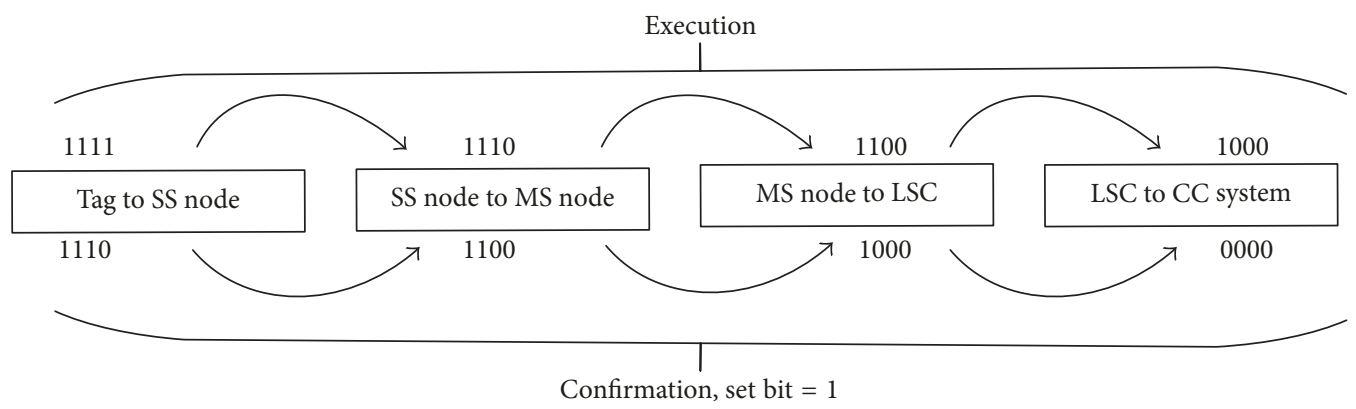

FIGURE 14: Vacant parking lot: reading information flow.

and nonvacant parking lots information is displayed which is measured individually from the parking points through the sensor nodes.

In Table 4, the randomly six parking points using Figure 9, that is, PP01, PP02, PP18, PP17, PP12, and PP15 $\epsilon$ $(\mathrm{PP})_{i}$, are selected among others and the status for the numerous parking lots is observed. However, the numerous parking lots are also randomly chosen from the selective six parking points. Thus, the status whether the parking lots are vacant $=1$ or nonvacant $=0$ in parking points is observed directly from the sensor nodes installed. For the system users, this information is very important, with which they are able to make parking reservations. Upon user selection for parking point, the information simultaneously displays the status of the parking lots; therefore the user has awareness about the parking lots status and, of course, saves time that might be wasted during searching for parking lots' availability.

As mentioned, the SVPS system is efficient in its overall processing, such as in parking searching and reservations and others; thereby the numerous of parking lots in parking points $(\mathrm{PP})_{(i \leq k)}$ are equipped with the sensor nodes. The networked master-slave-sensor nodes are entirely controlled and managed through the CC system, in cases of command or information write operation and read operation, and are fully functional to get or transmit information. As soon, the slave-sensor node gets the vehicle information through its embedded RFID tag, the CC system will also get the same computed information in minimal time session. However, the time always varies in cases where the CC system gets/reads information and instructs/writes command, from/to the sensor nodes. For each transaction, that is, write and read, the total time that required completing the process

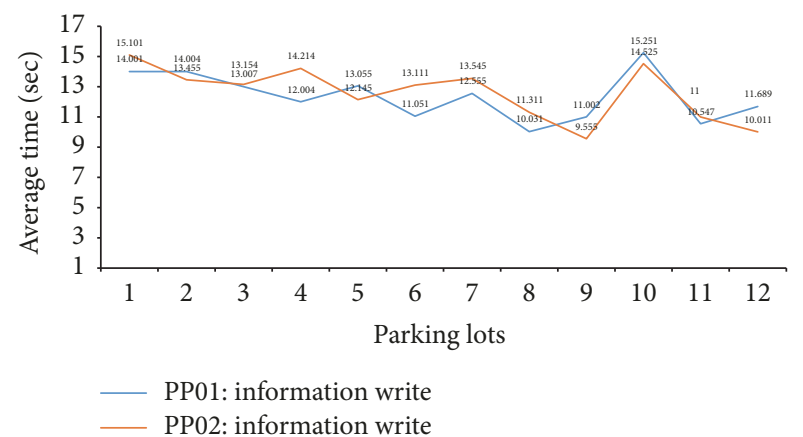

FIGURE 15: Average time computation during information writing.

is efficiently observed by the system. For that, in Figures 15-18, few successful experiments are conducted to compute the total time required during performing the write and read operations. The computed time in each experiment is average computed time in seconds and the overall computed results are considered as the most appropriate measurements according to our best knowledge. Further, the measurements are conducted using simulation, without any real equipment used, running in a computer machine Intel Core i7 system. Therefore, overall results conducted are nearly assumed to be average and accurate results. The average times in Figures 15-18 are measured by CC system input commands many times, and for these measurements, simulation continuously run about 5880 seconds.

In Figures 15 and 16, 12 experiments are randomly selected and performed to get the total average time while the CC system sent information (i.e., write commands) to and got 
TABLE 4: Reading operation and computation.

\begin{tabular}{|c|c|c|c|c|c|c|c|c|c|c|c|}
\hline \multicolumn{12}{|c|}{ Parking lots status $($ vacant $=1 ;$ nonvacant $=0)$} \\
\hline \multicolumn{2}{|c|}{ PP01 } & \multicolumn{2}{|c|}{ PP02 } & \multicolumn{2}{|c|}{ PP18 } & \multicolumn{2}{|c|}{ PP17 } & \multicolumn{2}{|c|}{ PP12 } & \multicolumn{2}{|c|}{ PP15 } \\
\hline LP001 & 0 & LP001 & 1 & LP001 & 0 & LP001 & 1 & LP001 & 0 & LP001 & 1 \\
\hline LP002 & 0 & LP002 & 0 & LP002 & 1 & LP002 & 1 & LP002 & 0 & LP002 & 0 \\
\hline LP003 & 0 & LP003 & 0 & LP003 & 1 & LP003 & 1 & LP003 & 0 & LP003 & 0 \\
\hline LP004 & 1 & LP004 & 1 & LP004 & 1 & LP004 & 1 & LP004 & 0 & LP004 & 1 \\
\hline LP005 & 0 & LP005 & 0 & LP005 & 1 & LP005 & 1 & LP005 & 0 & LP005 & 1 \\
\hline LP006 & 0 & LP006 & 1 & LP006 & 1 & LP006 & 1 & LP006 & 0 & LP006 & 1 \\
\hline LP007 & 1 & LP007 & 0 & LP007 & 1 & LP007 & 1 & LP007 & 0 & LP007 & 1 \\
\hline LP008 & 1 & LP008 & 0 & LP008 & 1 & LP008 & 1 & LP008 & 0 & LP008 & 1 \\
\hline LP009 & 1 & LP009 & 0 & LP009 & 0 & LP009 & 1 & LP009 & 0 & LP009 & 1 \\
\hline LP010 & 0 & LP010 & 1 & LP010 & 1 & LP010 & 1 & LP010 & 0 & LP010 & 1 \\
\hline LP011 & 1 & LP011 & 1 & LP011 & 1 & LP011 & 1 & LP011 & 0 & LP011 & 1 \\
\hline LP012 & 0 & LP012 & 1 & LP012 & 1 & LP012 & 1 & LP012 & 0 & LP012 & 1 \\
\hline LP013 & 1 & LP013 & 1 & LP013 & 1 & LP013 & 1 & LP013 & 0 & LP013 & 1 \\
\hline LP014 & 0 & LP014 & 1 & LP014 & 1 & LP014 & 1 & LP014 & 0 & LP014 & 1 \\
\hline LP015 & 0 & LP015 & 1 & LP015 & 1 & LP015 & 1 & LP015 & 0 & LP015 & 1 \\
\hline LP016 & 0 & LP016 & 1 & LP016 & 1 & LP016 & 1 & LP016 & 0 & LP016 & 1 \\
\hline LP017 & 1 & LP017 & 1 & LP017 & 1 & LP017 & 1 & LP017 & 0 & LP017 & 1 \\
\hline LP018 & 0 & LP018 & 0 & LP018 & 1 & LP018 & 1 & LP018 & 0 & LP018 & 1 \\
\hline LP019 & 0 & LP019 & 1 & LP019 & 1 & LP019 & 1 & LP019 & 0 & LP019 & 1 \\
\hline LP020 & 0 & LP020 & 1 & LP020 & 1 & LP020 & 1 & LP020 & 0 & LP020 & 1 \\
\hline LP021 & 0 & LP021 & 1 & LP021 & 0 & LP021 & 1 & LP021 & 0 & LP021 & 1 \\
\hline LP022 & 0 & LP022 & 1 & LP022 & 1 & LP022 & 1 & LP022 & 0 & LP022 & 1 \\
\hline LP023 & 1 & LP023 & 1 & LP023 & 1 & LP023 & 1 & LP023 & 0 & LP023 & 1 \\
\hline LP024 & 0 & LP024 & 1 & LP024 & 0 & LP024 & 1 & LP024 & 0 & LP024 & 1 \\
\hline$\vdots$ & $\vdots$ & $\vdots$ & $\vdots$ & $\vdots$ & $\vdots$ & $\vdots$ & $\vdots$ & $\vdots$ & $\vdots$ & $\vdots$ & $\vdots$ \\
\hline LP120 & 0 & LP120 & 1 & LP115 & 0 & LP145 & 1 & LP090 & 0 & LP140 & 1 \\
\hline
\end{tabular}

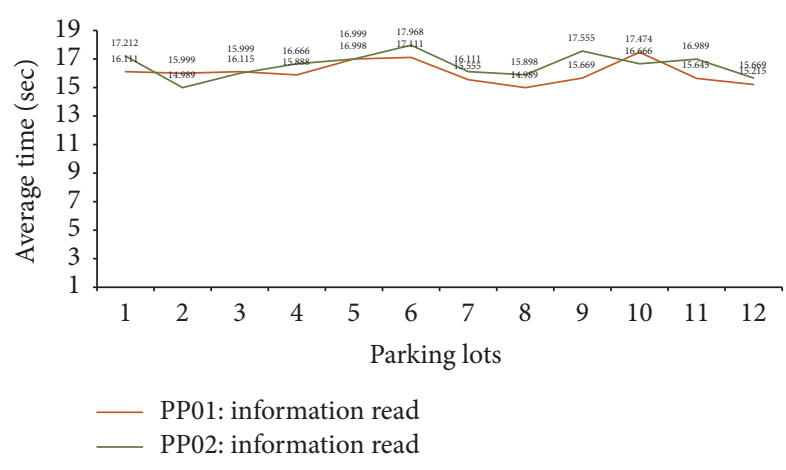

FIGURE 16: Average time computation during information reading.

a response (i.e., read commands) from the sensor nodes networked in the parking points PP01 and PP02. Similarly, in Figures 17 and 18, the total average time is observed in 12 experiments, individually conducted, while information is sent to and received from sensor nodes networked in the parking points PP18, PP17, PP12, and PP15.

\section{Conclusion and Future Work}

The proposed study or SVPS system has been contributed to developing a more advanced and smart parking system, and to overcome the problematics of vehicle parking (during parking searching) in a metropolitan city mostly today's parking systems have. For the smart parking, SVPS system has been contributed to fulfilling several objectives, that is, parking searching and reservations, which are the key requirements of today's smart parking system. In spite of the other services provided, the parking searching and reservation module is one of the important modules which provides efficient ways to do searching for the parking points using SVPS-Map facility available online through login into the accounts provided by the system for the registered users. Therefore, users are able to do searching for the parking points, according to their interest, having the indications of vacant or nonvacant parking lots to perform further parking reservations. As a result, users could save enough time that is mostly wasted in searching for parking and the parking lots availability prior to the final reservations. For information computation, the SVPS system 


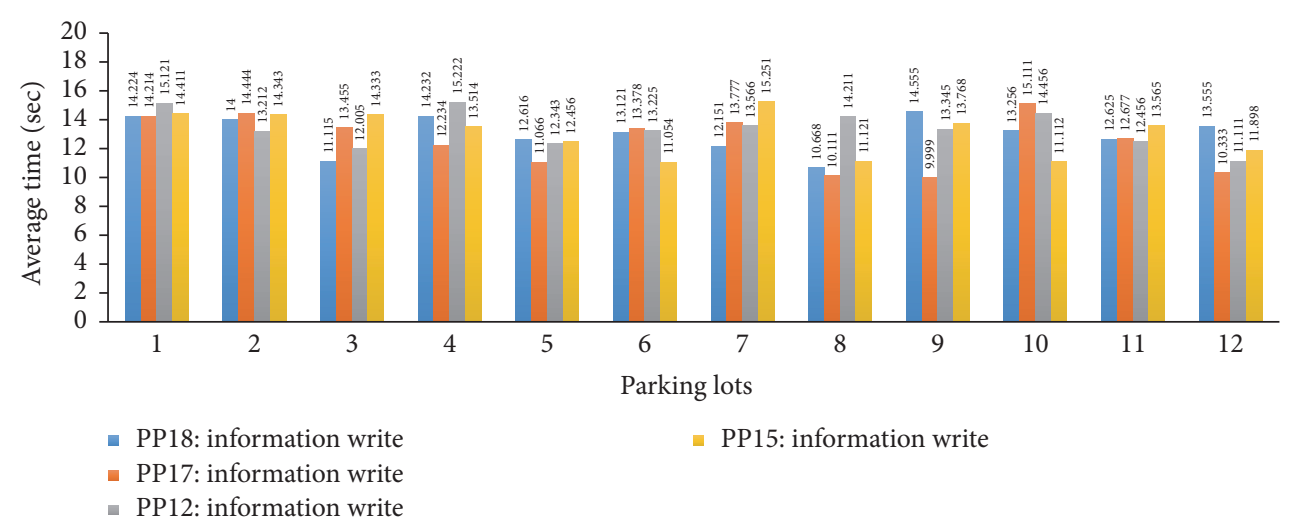

FIGURE 17: Selective parking points and information writing.

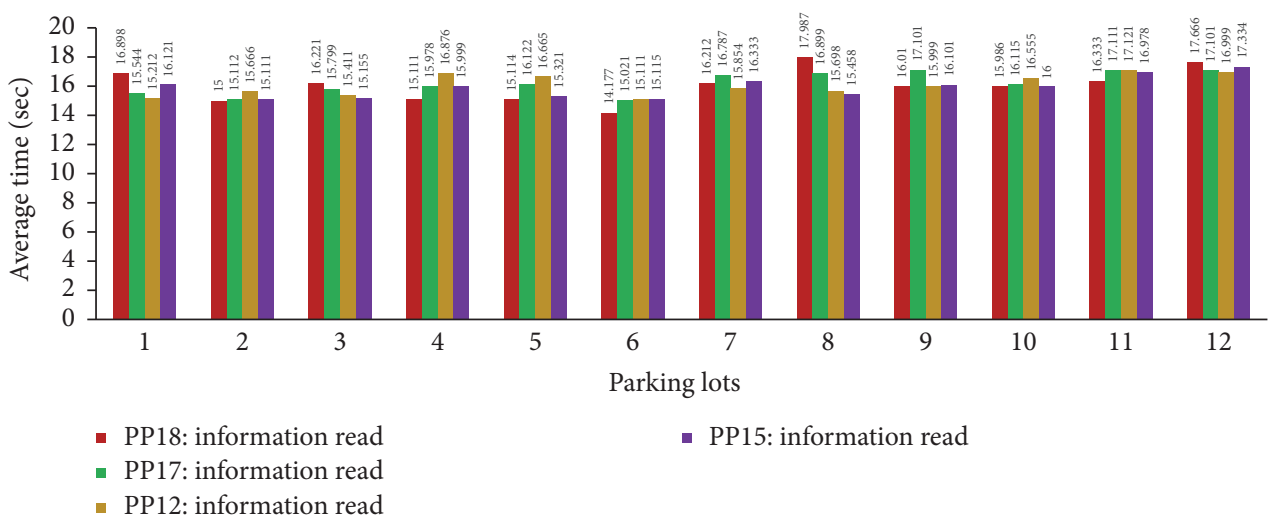

FIGURE 18: Selective parking points and information reading.

employed advanced integrated RFID and WSN or RFIDWSN technology to network all its parking points which are fully set up with the sensor nodes; therefore each time the RFID tags read the information, for example, vehicle checkin and checkout information, transmitted to the CC system. Overall the information either read or write, the instructions, or commands are always inputted by the CC system.

This study is rigorous in its current development stages and for the further future enhancements, the current study aims at the vehicle parking reservations, resource management, and remote monitoring. In future, therefore the important modules, such as (1) indoor parking interactive mapping, (2) the external and internal system security design, and (3) secure-application access for smart cellular devices, will be designed and implemented as parts of SVPS system.

\section{Conflicts of Interest}

The authors declare that there are no conflicts of interest regarding the publication of this paper.

\section{Acknowledgments}

This work was supported by the Faculty Research Fund of Sejong University in year 2017, Institute for Information \& Communications Technology Promotion (IITP) grant funded by the Korea Government (MSIT) (no. 2017-0-00756, Development of Interoperability and Management Technology of IoT System with Heterogeneous ID Mechanism), Next-Generation Information Computing Development Program through the National Research Foundation of Korea (NRF) funded by the Ministry of Science and ICT (NRF2014M3C4A7030503), and the National Research Foundation of Korea (NRF) granted by the Korea Government (MSP) (no. 2017R1A2B4006667).

\section{References}

[1] T. N. Pham, M.-F. Tsai, D. B. Nguyen, C.-R. Dow, and D.J. Deng, "A Cloud-Based Smart-Parking System Based on Internet-of-Things Technologies," IEEE Access, vol. 3, pp. 15811591, 2015.

[2] A. Khanna and R. Anand, "IoT based smart parking system," in Proceedings of the 2016 International Conference on Internet of Things and Applications, IOTA 2016, pp. 266-270, Pune, India, June 2016.

[3] C. Rhodes, W. Blewitt, C. Sharp, G. Ushaw, and G. Morgan, "Smart routing: A novel application of collaborative pathfinding to smart parking systems," in Proceedings of the 16th IEEE Conference on Business Informatics, CBI 2014, pp. 119-126, Geneva, Switzerland, July 2014.

[4] N. Mejri, M. Ayari, R. Langar, F. Kamoun, G. Pujolle, and L. Saidane, "Cooperation versus competition towards an efficient 
parking assignment solution," in Proceedings of the 2014 1st IEEE International Conference on Communications, ICC 2014, pp. 2915-2920, Sydney, Australia, June 2014.

[5] C. J. Rodier and S. A. Shaheen, "Transit-based smart parking: An evaluation of the San Francisco Bay area field test," Transportation Research Part C: Emerging Technologies, vol. 18, no. 2, pp. 225-233, 2010.

[6] A. Bagula, L. Castelli, and M. Zennaro, "On the design of smart parking networks in the smart cities: An optimal sensor placement model," Sensors, vol. 15, no. 7, pp. 15443-15467, 2015.

[7] Z. Pala and N. Inanç, "Smart parking applications using RFID technology," in Proceedings of the 1st Annual RFID Eurasia, pp. 1-3, Istanbul, Turkey, September 2007.

[8] R. Want, "An introduction to RFID technology," IEEE Pervasive Computing, vol. 5, no. 1, pp. 25-33, 2006.

[9] J. Majrouhi Sardroud, "Influence of RFID technology on automated management of construction materials and components," Scientia Iranica, vol. 19, no. 3, pp. 381-392, 2012.

[10] L. Mainetti, L. Palano, L. Patrono, M. L. Stefanizzi, and R. Vergallo, "Integration of RFID and WSN technologies in a Smart Parking System," in Proceedings of the 22nd International Conference on Software, Telecommunications and Computer Networks, SoftCOM 2014, pp. 104-110, Split, Croatia, September 2014.

[11] Pala, Zeydin, and N. Inanc, "Utilizing RFID for smart parking applications," Facta universitatis-series: Mechanical Engineering, vol. 7, no. 1, pp. 101-118, 2009.

[12] K. Hassoune, W. Dachry, F. Moutaouakkil, and H. Medromi, "Smart parking systems: A survey," in Proceedings of the 11th International Conference on Intelligent Systems: Theories and Applications, SITA 2016, pp. 1-6, Mohammedia, Morocco, October 2016.

[13] C. W. Hsu, M. H. Shih, H. Y. Huang, Y. C. Shiue, and S. C. Huang, "Verification of smart guiding system to search for parking space via DSRC communication," in Proceedings of the 2012 12th International Conference on ITS Telecommunications, ITST 2012, Taipei, Taiwan, November 2012.

[14] M. Du, J. Fang, and H. Cao, "A new solution for city parking guiding based on Internet of Things and multi-level multiagent," in Proceedings of the 2011 International Conference on Electronics, Communications and Control, ICECC 2011, pp. 4093-4096, Ningbo, China, September 2011.

[15] L. Lambrinos and A. Dosis, "DisAssist: An internet of things and mobile communications platform for disabled parking space management," in Proceedings of the 2013 IEEE Global Communications Conference, GLOBECOM 2013, pp. 2810-2815, Atlanta, GA, USA, December 2013.

[16] H. Wang and W. He, "A reservation-based smart parking system," in Proceedings of the 2011 IEEE Conference on Computer Communications Workshops, INFOCOM WKSHPS 2011, Shanghai, China, April 2011.

[17] Y. Geng and C. G. Cassandras, "A new "Smart Parking" System Infrastructure and Implementation," Social and Behavioral Sciences, vol. 54, pp. 1278-1287, 2012.

[18] S. Qin and X. Yao, "An intelligent parking system based on GSM module," Applied Mathematics \& Information Sciences, vol. 7, no. 1L, pp. 55-59, 2013.

[19] R. E. Barone, T. Giuffre, S. M. Siniscalchi, M. A. Morgano, and G. Tesoriere, "Architecture for parking management in smart cities," IET Intelligent Transport Systems, vol. 8, no. 5, pp. 445452, August 2014.
[20] S. A. Faheem, G. M. Mahmud, M. Khan, H. Rahman, and Zafar, "A Survey of Intelligent Car Parking System," Journal of Applied Research and Technology, vol. 11, no. 5, pp. 714-726, 2013.

[21] F. I. Shaikh, P. N. Jadhav, S. P. Bandarkar et al., "Smart Parking System Based on Embedded System and Sensor Network," International Journal of Computer Applications, vol. 140, no. 12, 2016.

[22] A. Correa, G. Boquet, A. Morell, and etal., "Autonomous car parking system through a cooperative vehicular positioning network," Sensors, vol. 17, no. 4, article no. 848, 2017.

[23] S. Cui, M. Wu, C. Liu, and N. Rong, "The research and implement of the intelligent parking reservation management system based on ZigBee technology," in Proceedings of the 2014 6th International Conference on Measuring Technology and Mechatronics Automation, ICMTMA 2014, pp. 741-744, Zhangjiajie, China, January 2014.

[24] D. J. Bonde, R. S. Shende, A. S. Kedari, K. S. Gaikwad, and A. U. Bhokre, "Automated car parking system commanded by Android application," in Proceedings of the 4th International Conference on Computer Communication and Informatics (ICCCI '14), pp. 1-4, IEEE, Coimbatore, India, January 2014.

[25] M. O. Reza et al., "Smart parking system with image processing facility," International Journal of Intelligent Systems and Applications, vol. 4, no. 3, pp. 41-47, 2012.

[26] M. Y. I. Idris, E. M. Tamil, Z. Razak, N. M. Noor, and L. W. Km, "Smart parking system using image processing techniques in wireless sensor network environment," Information Technology Journal, vol. 8, no. 2, pp. 114-127, 2009.

[27] S. Banerjee, P. Choudekar, and M. K. Muju, "Real time car parking system using image processing," in Proceedings of the 2011 3rd International Conference on Electronics Computer Technology, ICECT 2011, pp. 99-103, Kanyakumari, India, April 2011.

[28] I. Song, K. Gowan, J. Nery et al., "Intelligent parking system design using FPGA," in Proceedings of the 2006 International Conference on Field Programmable Logic and Applications, FPL, pp. 1-6, Madrid, Spain, August 2006.

[29] M. Gallo, L. D’Acierno, and B. Montella, "A multilayer model to simulate cruising for parking in urban areas," Transport Policy, vol. 18, no. 5, pp. 735-744, 2011.

[30] D. Van der Goot, "A model to describe the choice of parking places," Transportation Research Part A: General, vol. 16, no. 2, pp. 109-115, 1982.

[31] R. G. Thompson and A. J. Richardson, "A parking search model," Transportation Research Part A: Policy and Practice, vol. 32, no. 3, pp. 159-170, 1998.

[32] W. Young and M. Taylor, "A parking model hierarchy," Transportation, vol. 18, no. 1, pp. 37-58, 1991.

[33] M. M. Berenger Vianna, L. da Silva Portugal, and R. Balassiano, "Intelligent transportation systems and parking management: Implementation potential in a Brazilian city," Cities, vol. 21, no. 2, pp. 137-148, 2004.

[34] J. Li, H. Hu, Q. Ke, and N. Xiong, "A novel topology linkcontrolling approach for active defense of a node in a network," Sensors, vol. 17, no. 3, article no. 553, 2017.

[35] P.-F. Wu, F. Xiao, C. Sha, H.-P. Huang, R.-C. Wang, and N.$\mathrm{X}$. Xiong, "Node scheduling strategies for achieving full-view area coverage in camera sensor networks," Sensors, vol. 17, no. 6, article no. 1303, 2017.

[36] H. Cheng, Y. Chen, N. Xiong, and F. Li, "Layer-based data aggregation and performance analysis in wireless sensor networks," 
Journal of Applied Mathematics, vol. 2013, Article ID 502381, 2013.

[37] F. Xia, R. Hao, J. Li, N. Xiong, L. T. Yang, and Y. Zhang, "Adaptive GTS allocation in IEEE 802.15.4 for real-time wireless sensor networks," Journal of Systems Architecture, vol. 59, no. 10, pp. 1231-1242, 2013.

[38] Roussos and George, Networked RFID: systems, software and services, Springer Science \& Business Media, 2008.

[39] J. P. Benson, T. O’Donovan, P. O’Sullivan et al., "Car-park management using wireless sensor networks," in Proceedings of the 31st Annual IEEE Conference on Local Computer Networks, LCN 2006, pp. 588-595, Tampa, FL, USA, November 2006.

[40] Shaheen and Susan, Smart parking management field test: A bay area rapid transit (bart) district parking demonstration, Institute of Transportation Studies, 2005.

[41] R. Yusnita, F. Norbaya, and N. Basharuddin, "Intelligent Parking Space Detection System Based on Image Processing," International Journal of Innovation, Management and Technology, vol. 3, article no. 232, no. 3, 2012.

[42] P. E. Hart, N. J. Nilsson, and B. Raphael, "A formal basis for the heuristic determination of minimum cost paths," IEEE Transactions on Systems, Man, and Cybernetics, vol. 4, no. 2, pp. 100-107, 1968.

[43] M. M. Rashid, A. Musa, M. A. Rahman, N. Farahana, and A. Farhana, "Automatic parking management system and parking fee collection based on number plate recognition," International Journal of Machine Learning and Computing, vol. 2, no. 2, pp. 93-98, 2012.

[44] H. R. H. Al-Absi, J. D. D. Devaraj, P. Sebastian, and Y. V. Voon, "Vision-based automated parking system," in Proceedings of the 10th International Conference on Information Sciences, Signal Processing and their Applications, ISSPA 2010, pp. 757760, Kuala Lumpur, Malaysia, May 2010.

[45] E. Karbab, D. Djenouri, S. Boulkaboul, and A. Bagula, "Car park management with networked wireless sensors and active RFID," in Proceedings of the IEEE International Conference on Electro/Information Technology (EIT '15), pp. 373-378, IEEE, Dekalb, Ga, USA, May 2015.

[46] E. P. C. EPCglobal, "Radio-frequency identity protocols class1 generation-2 uhf rfid protocol for communications at 860 mhz-960 mhz version 1.0. 9," 2004, K. Chiew et al./On False Authenticationsfor C1G2 Passive RFID Tags 65.

[47] C. Ying and Z. Fu-Hong, "A system design for UHF RFID reader," in Proceedings of the 2008 11th IEEE International Conference on Communication Technology, ICCT 2008, pp. 301304, Hangzhou, China, November 2008.

[48] A. Shahzad, M. Lee, H. D. Kim, S.-M. Woo, and N. Xiong, "New security development and trends to secure the SCADA sensors automated transmission during critical sessions," Symmetry, vol. 7, no. 4, pp. 1945-1980, 2015.

[49] A. Shahzad, M. Lee, C. Lee et al., "The protocol design and New approach for SCADA security enhancement during sensors broadcasting system," Multimedia Tools and Applications, vol. 75, no. 22, pp. 14641-14668, 2016. 


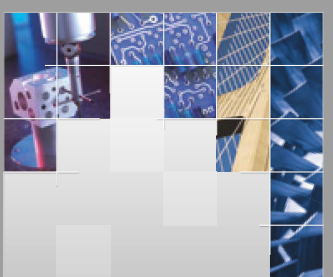

\section{Enfincering}
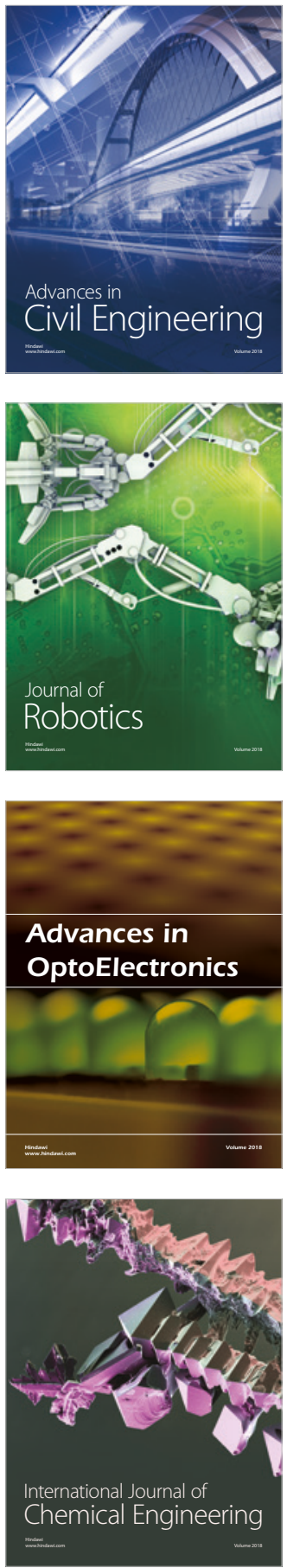

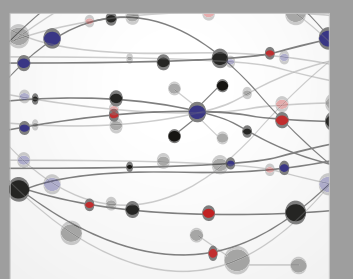

\section{Rotating \\ Machinery}

The Scientific World Journal

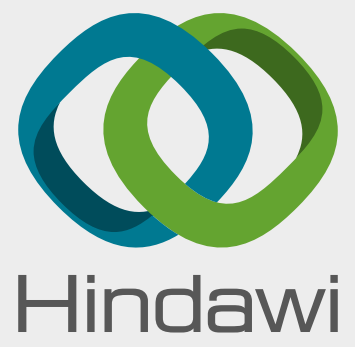

Submit your manuscripts at

www.hindawi.com
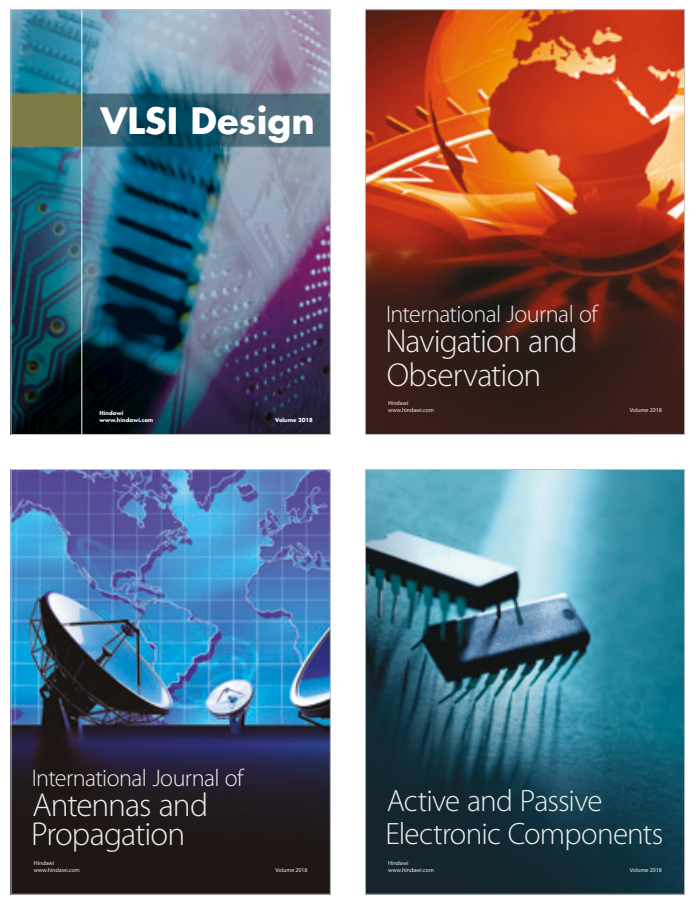
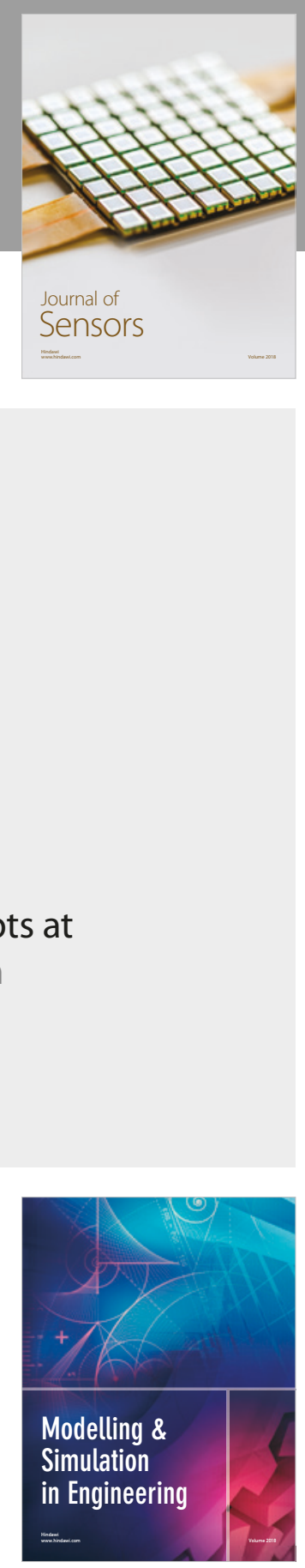

\section{Advances \\ Multimedia}
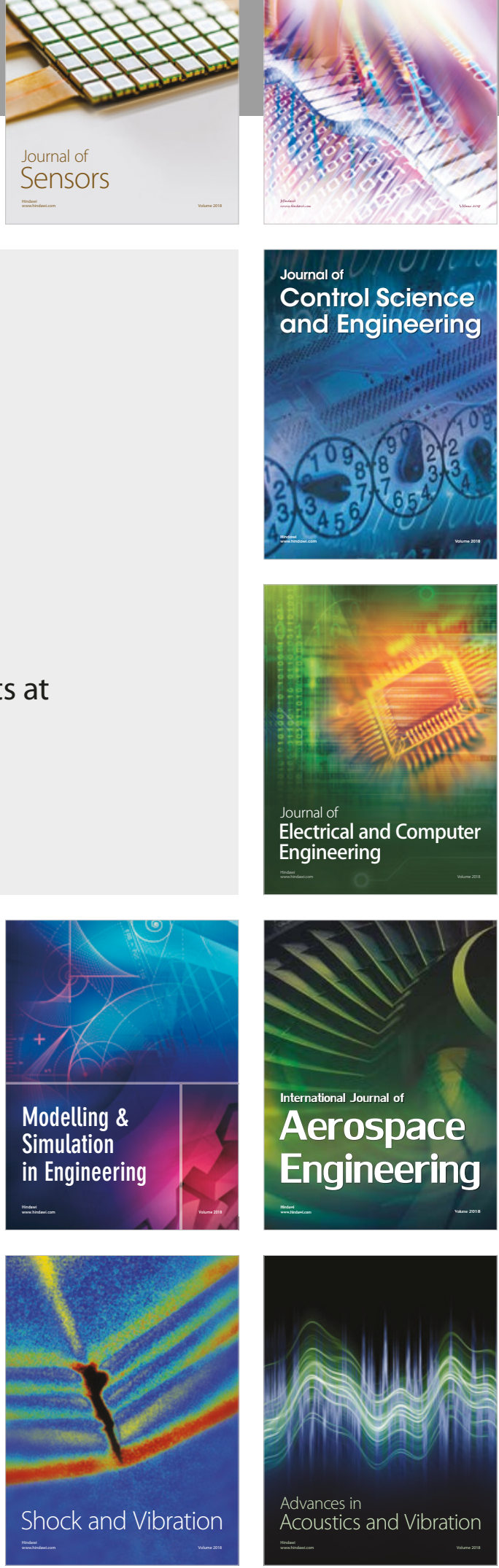